\title{
Vibration transmission across junctions of double walls using the wave approach and statistical energy analysis ${ }^{\text {tr }}$
}

\author{
A. Dijckmans* \\ KU Leuven, Department of Civil Engineering, Kasteelpark Arenberg 40, B-3001 Leuven, Belgium
}

\begin{abstract}
The sound insulation between adjacent rooms in buildings is not only determined by direct airborne sound transmission through the common wall, but also by structure-borne flanking transmission. To quantify the flanking transmission, the determination of the vibrational energy transmission at a junction between plates is crucial. In this paper, statistical models are developed for the prediction of flanking transmission across rigid junctions composed of single and double walls. The coupling loss factors are determined from wave theory for semi-infinite plates under the assumption of diffuse vibration fields. In-plane wave transmission must be accounted for in the case of wave transmission across double wall junctions. In general, the vibration transmission across double walls shows similar trends as the vibration transmission across single walls. An initial assessment of flanking transmission across a double wall junction can therefore be made by use of an equivalent single wall junction model. The developed models can also be used to investigate structure-borne flanking transmission across two adjacent single wall junctions. It is shown that indirect coupling between non-adjacent plates is important and should be accounted for in statistical models.
\end{abstract}

Keywords: sound insulation, flanking, double walls, statistical energy analysis

\section{Introduction}

The prediction of the sound insulation between rooms is a complex problem, as not only direct transmission, but also structure-borne flanking transmission plays a role. The International Standards ISO 15712-1/2 [1] and European Standards EN 12354-1/2 [2] use a simplified approach based on Statistical Energy Analysis

\footnotetext{
The archived file is not the final published version of the article.

A. Dijckmans. Vibration transmission across junctions of double walls using the wave approach and statistical energy analysis. Acta Acustica united with Acustica, 102(3):488-502, 2016.

(C)(2016) S. Hirzel Verlag/European Acoustics Association

The definitive publisher-authenticated version is available online at http://www.ingentaconnect.com/content/dav/aaua http://dx.doi.org/10.3813/AAA.918967

Readers must contact the publisher for reprint or permission to use the material in any form.

*Corresponding author. Phone: + 32163777 96. Fax: + 3216321988.

Email address: arne.dijckmans@kuleuven.be (A. Dijckmans*)
} 
(a)
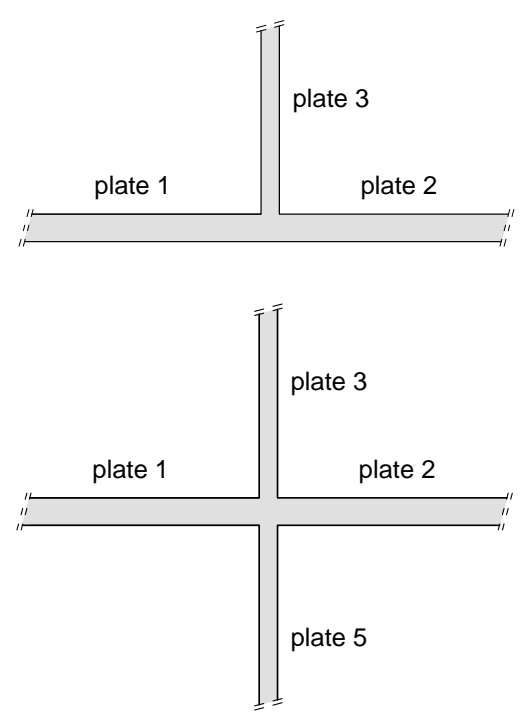

(c)

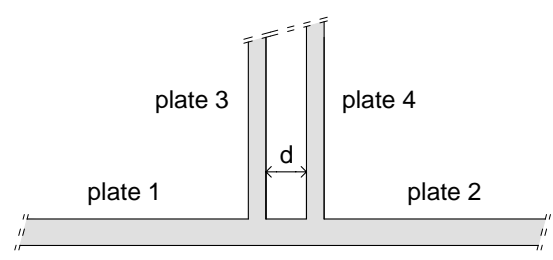

(b)

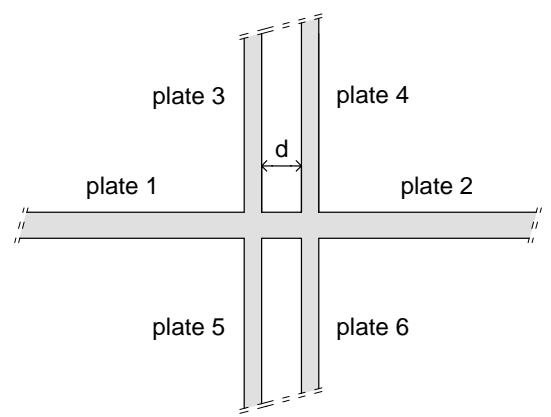

(d)

Figure 1: Geometry for (a) a single wall T-junction, (b) a double wall T-junction, (c) a single wall X-junction, and (d) a double wall X-junction.

(SEA) to model flanking transmission. An important input quantity for the models is the vibration reduction index $K_{i j}$, which describes the transmission of bending wave vibration across a junction of plates. For single plate junctions like shown in figures 1a and 1c, empirical models based on measurements [3] and simulations [4] are available. Currently, such models do not exist for junctions with double walls like shown in figures $1 \mathrm{~b}$ and $1 \mathrm{~d}$. Nevertheless, heavyweight cavity walls are commonly used as party wall between row houses and in apartment buildings in Belgium [5]. The concept of double leaf walls is also essential to provide the necessary sound insulation with lightweight constructions. Timber framed constructions are widely used for houses and there is an increased interest for timber framed apartment houses in view of sustainability concerns. For complex plate junctions with double leaf walls, one mainly has to rely on measured $K_{i j}$-data. Schoenwald $e t$ al. $[6,7]$ experimentally analyzed the vibration transmission across lightweight double leaf walls and solid wood double walls with continuous floors. Alternatively, one may use data for single wall junctions as a first estimate for a double wall junction. A double wall $\mathrm{T}$-junction (figure $1 \mathrm{~b}$ ) is often approximated by a single wall $\mathrm{X}$-junction (figure 1c). In [7], the measured data for a X-junction of double solid timber walls were compared with measurements of a single wall $\mathrm{X}$-junction, showing that the differences are limited. It was noted however that such estimates should be handled with care, as little is known about the general validity and accuracy of these single wall approximations. In this paper, the vibration transmission across double wall junctions is therefore analyzed in detail by means of analytical and statistical models based on the wave approach. 
The wave approach has been widely used to predict the structure-borne sound transmission between semi-infinite plates. The angular averaged transmission coefficients obtained from these calculations can be used in SEA models [8]. The most comprehensive overview on structure-borne sound is given by Cremer et al. [9]. The original calculation models $[10,11]$ for rigid, right-angled junctions have gradually been extended to more complicated junctions [12], including different wave types [13, 14] and thick plate effects [15]. Mees and Vermeir [16] extended the wave approach to single wall junctions with elastic interlayers. Furthermore, a hinge connection was introduced to model incomplete contact between plates which results in a negligible transfer of bending moments. Bosmans et al. [17, 18] investigated structure-borne sound transmission across junctions of orthotropic plates [17] and junctions with point connections [18]. More recently, Hopkins [4] used classic wave theory for vibration transmission across rigid junctions in heavyweight buildings built from solid masonry or concrete walls and floors. He showed that the contribution of in-plane waves can be important at mid and high frequencies.

Most contributions in literature deal with single wall junctions. In this paper, it is the aim to apply the wave approach to double wall junctions as commonly encountered in buildings in Belgium. The junctions of figures $1 \mathrm{~b}$ and $1 \mathrm{~d}$ are modeled by two line junctions which are coupled to each other by a plate with length equal to the cavity width of the double wall. Similar semi-infinite plate models with strip plates, i.e. finite width plates that meet at more than one line junction, have been used to describe structural transmission by line connections in double walls [19, 20]. Bhattacharya et al. [19] investigated wave transmission via a strip plate in a double wall junction for a bending wave incident at normal incidence, taking into account bending waves and in-plane quasi-longitudinal waves. They showed that a significant amount of energy is transported by quasi-longitudinal waves in the coupling plate. Craik et al. [20] generalized the model of Bhattacharya et al. [19] to angular average transmission coefficients including bending, quasi-longitudinal and in-plane shear wave motion. This model was also compared with simplified models where the line connection between the two parallel plates was either modeled as a beam or as a separate SEA subsystem [20]. The finite width plate model gave the best agreement with measurement results for a freely suspended, lightweight H-structure. Previously, Craik et al. [21, 22] have used simplified approaches to model structureborne transmission between double walls by treating the line connection as a massless and rigid beam [21] or as a rotational spring [22]. Gibbs [23] developed a general model of bending and in-plane vibration of coupled plates where there is more than one junction. The model was applied to a series of parallel $\mathrm{T}$-junctions to show the influence of resonances in the coupling plates and the fact that in-plane vibration can travel appreciable distances through several junctions.

SEA models using the angular average transmission coefficient determined from wave theory tend to give good agreement with measurements and numerical modeling when the modal overlap factor is greater than or equal to unity $[4,24]$ and when the mode count within a frequency band is at least five [25]. Heavyweight walls and floors often have low modal overlap and low modal density in a broad frequency 
range. Because these structures are highly damped when fully connected in real buildings, SEA can give reasonable results even when these criteria are not met $[8,26,27]$. Furthermore, a preliminary comparison with numerical simulations for structure-borne sound transmission across single and double concrete wall junctions has indicated that SEA can give good insight in the general trends [28]. In this paper, SEA is therefore applied to investigate flanking transmission across rigid junctions consisting of both lightweight and heavyweight walls. Section 2 describes the wave transmission between semi-infinite plates in detail. The model of Bosmans [29] for junctions of single plates is extended to the case of double walls. Transmission coefficients for double wall junctions are compared with those of single wall junctions and the influence of in-plane wave motion is investigated. In section 3, velocity level differences between junctions built-up of finite plates as calculated with SEA are presented for double wall junctions. Furthermore, the applicability of equivalent single wall junction models is assessed and a limit case of two parallel single wall junctions is investigated. Conclusions are given in section 4 .

\section{Wave transmission between semi-infinite plates}

The wave approach is based on the interaction of semi-infinite plates, where the plates are assumed to intersect along infinite joints. The excitation of the plate assembly is taken as a plane wave incident upon the junction on one of the plates. The incident wave generates bending, quasi-longitudinal and in-plane transverse shear waves propagating away from the junction on all plates. The forces and displacements at the plate edges are expressed in terms of the amplitudes of these propagating waves. The equilibrium and continuity conditions at the junction lines lead to a set of linear equations, the solution of which yields the unknown wave amplitudes. The energy flow associated with the traveling waves is determined by their amplitudes. Finally, structure-borne sound transmission is quantified by the transmission coefficient, which is defined as the ratio of the transmitted intensity to the intensity carried by the incident wave.

\subsection{Problem definition and principles of the wave approach}

The model is restricted to junctions of single or double plates connected along the entire width of a common edge. Figures $1 \mathrm{~b}$ and $1 \mathrm{~d}$ show examples of a double wall $\mathrm{T}$-junction and a double wall $\mathrm{X}$-junction, respectively. The plates are assumed solid, homogeneous and isotropic, and are modeled using thin plate theory. Airborne coupling between the double wall leafs is neglected. For lightweight double walls, the airborne coupling can be dominant, especially below and around the mass-spring-mass resonance frequency. In this case, a comparison with measured vibration reduction indices that include the airborne path should be made with caution. For heavyweight cavity walls, the structure-borne transmission is however most important in the entire frequency range of interest.

An example junction is shown in figure 2 where a double wall T-junction is considered. The model uses two junction beams to connect the plates together, but the beams do not represent a physical part of 


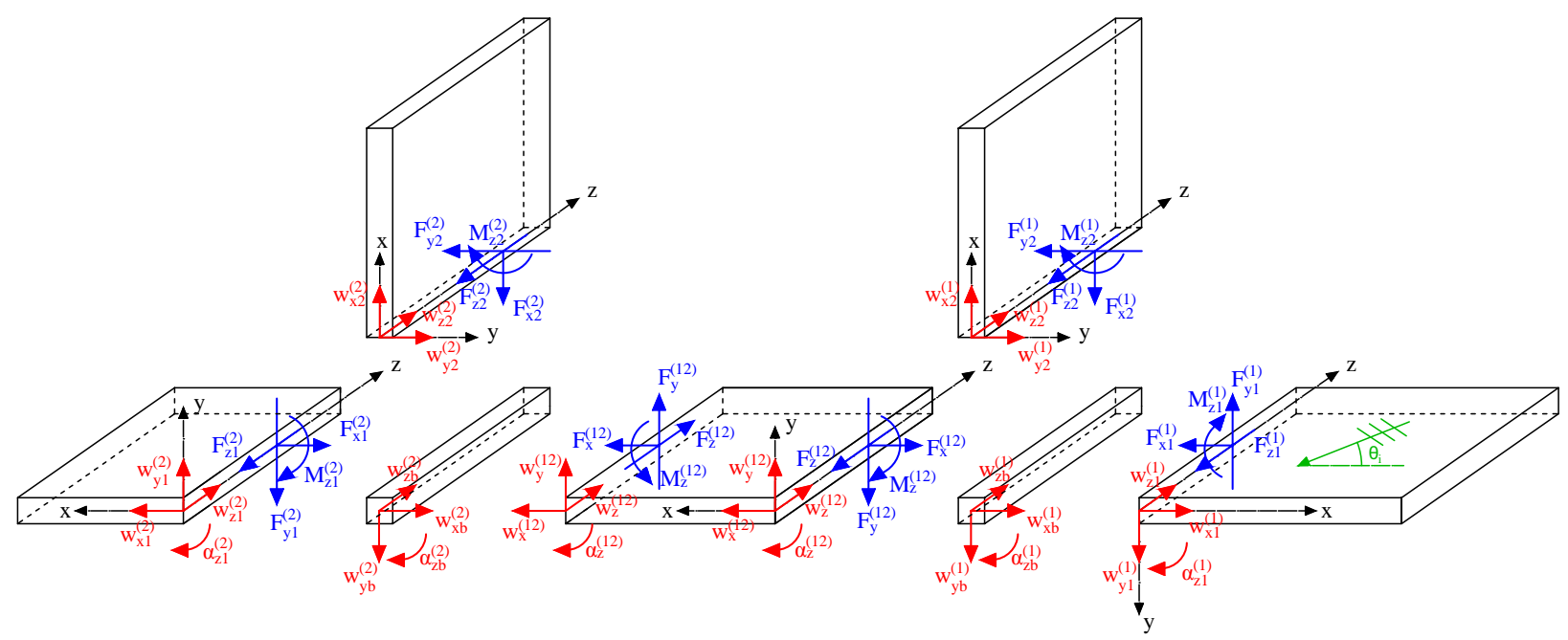

Figure 2: Exploded view of a double wall T-junction $\left(N^{(1)}=2, N^{(2)}=2, \theta_{1}^{(1)}=0^{\circ}, \theta_{2}^{(1)}=270^{\circ}, \theta_{1}^{(2)}=180^{\circ}, \theta_{2}^{(2)}=270^{\circ}\right.$, $\left.\theta^{(12)}=180^{\circ}\right)$ with variables used to describe wave motion.

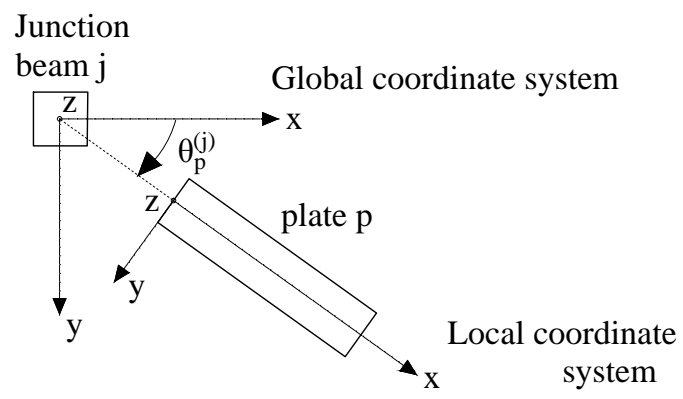

Figure 3: Global and local coordinate system for each plate $p$ connected to junction beam $j$.

the real junction. The beams are free to rotate and to undergo displacement in the three directions. The junction beams are coupled to each other by a finite length plate. The length of this coupling plate is equal to the cavity width of the double wall. In the present case, two semi-infinite plates are connected to the first junction beam and two semi-infinite plates to the second junction beam. The derivation however is general and equally applies to other double wall junctions with different number of plates connected to the two junction beams. The number of plates connected to junction beam $j$, not counting the coupling plate, is denoted by $N^{(j)}$. The variables related to the coupling plate are indicated by the superscript (12). Variables related to plate $p$ of junction beam $j$ are indicated by a subscript $p$ and a superscript $(j)$. The coordinate systems to describe each plate and their position relative to the junction beam are shown in figure 3 . Wave motion on each plate is described using a local coordinate system where each plate lies in the $x z$ plane. Each plate that forms the junction is then connected to the junction beams using the global coordinate system.

The plate response is described by three harmonic wave types: bending, quasi-longitudinal and in-plane 
transverse shear waves. The transverse plate displacement $w_{y}$ fulfils Kirchhoff's thin plate bending wave equation:

$$
\nabla^{4} w_{y}-k_{\mathrm{B}}^{4} w_{y}=0
$$

where the bending wave number $k_{\mathrm{B}}$ and the plate bending stiffness $B^{\prime}$ per unit width are defined as

$$
\begin{aligned}
k_{\mathrm{B}} & =\sqrt[4]{\frac{m^{\prime \prime} \omega^{2}}{B^{\prime}}}, \\
B^{\prime} & =\frac{\underline{E} h^{3}}{12\left(1-\nu^{2}\right)} .
\end{aligned}
$$

with $m^{\prime \prime}=\rho h$ the surface mass density, $h$ the plate thickness, $\rho$ the density, $\underline{E}=E(1+j \eta)$ the complex Young's modulus, $\eta$ the loss factor, and $\nu$ the Poisson's ratio. The transverse displacement $w_{y}$ leads to a rotation about the $z$-axis, $\alpha_{z}$, a bending moment per unit width, $M_{z}$, and a shear force per unit width, $F_{y}$ (figure 2):

$$
\begin{aligned}
\alpha_{z} & =\frac{\partial w_{y}}{\partial x} \\
F_{y} & =-B^{\prime}\left(\frac{\partial^{3} w_{y}}{\partial x^{3}}+(2-\nu) \frac{\partial^{3} w_{y}}{\partial x \partial z^{2}}\right), \\
M_{z} & =-B^{\prime}\left(\frac{\partial^{2} w_{y}}{\partial x^{2}}+\nu \frac{\partial^{2} w_{y}}{\partial z^{2}}\right) .
\end{aligned}
$$

The in-plane displacements, $w_{x}$ and $w_{z}$, are related by two coupled equations of motion [9, 26, 29]. These equations can be uncoupled by introduction of a potential, $\Phi$, and a stream function, $\Psi$, which are related to the in-plane displacements as follows:

$$
\begin{aligned}
& w_{x}=\frac{\partial \Phi}{\partial x}-\frac{\partial \Psi}{\partial z} \\
& w_{z}=\frac{\partial \Phi}{\partial z}+\frac{\partial \Psi}{\partial x} .
\end{aligned}
$$

The equations of motion for in-plane quasi-longitudinal and transverse waves are then given by

$$
\begin{aligned}
& \nabla^{2} \Phi+k_{\mathrm{L}}^{2} \Phi=0 \\
& \nabla^{2} \Psi+k_{\mathrm{T}}^{2} \Psi=0,
\end{aligned}
$$

respectively. The quasi-longitudinal wave number $k_{\mathrm{L}}$ and transverse wave number $k_{\mathrm{T}}$ are given by

$$
\begin{aligned}
& k_{\mathrm{L}}=\omega \sqrt{\frac{\rho\left(1-\nu^{2}\right)}{\underline{E}}}, \\
& k_{\mathrm{T}}=\omega \sqrt{\frac{\rho}{\underline{G}}},
\end{aligned}
$$


with $\underline{G}=G(1+j \eta)$ the complex shear modulus. The in-plane waves give rise to a normal force per unit width, $F_{x}$, and an in-plane shear force per unit width, $F_{z}$ (figure 2):

$$
\begin{aligned}
F_{x} & =\frac{\underline{E} h}{1-\nu^{2}}\left(\frac{\partial w_{x}}{\partial x}+\nu \frac{\partial w_{z}}{\partial z}\right), \\
F_{z} & =\underline{G} h\left(\frac{\partial w_{x}}{\partial z}+\frac{\partial w_{z}}{\partial x}\right) .
\end{aligned}
$$

\subsection{Solutions to the equations of motion}

Incident waves can be bending, quasi-longitudinal, or transverse waves that travel in the negative $x$ direction towards the first junction at $x=0$ (local coordinates). For any incident wave with unit amplitude that impinges upon the junction at an angle of incidence, $\theta_{\mathrm{i}}$, the general form of the wave is

$$
e^{j k_{\mathrm{i}} x \cos \theta_{\mathrm{i}}} e^{-j k_{\mathrm{i}} z \sin \theta_{\mathrm{i}}}
$$

where $k_{\mathrm{i}}$ is the wavenumber. According to Snell's law, the waves generated at the junction have identical spatial dependence along the $z$-axis as imposed by the incident wave. The angle of incidence is therefore related to the angle of transmission $\theta_{\mathrm{t}}$ by

$$
k_{\mathrm{i}} \sin \theta_{\mathrm{i}}=k_{\mathrm{t}} \sin \theta_{\mathrm{t}},
$$

where $k_{\mathrm{t}}$ is the wavenumber of the transmitted wave. This gives the general form for transmitted waves as

$$
T e^{-j k_{x} x} e^{-j k_{\mathrm{i}} z \sin \theta_{\mathrm{i}}}
$$

where $T$ is the complex amplitude. Note that the time-dependence $e^{j \omega t}$ has been omitted for brevity. When $k_{\mathrm{i}}>k_{\mathrm{t}}$, there is a cut-off angle, $\theta_{\text {co }}$, above which there is no transmitted wave and the transmission coefficient is zero. This cut-off angle can be calculated from

$$
\theta_{\text {co }}=\arcsin \left(\frac{k_{\mathrm{t}}}{k_{\mathrm{i}}}\right)
$$

\subsubsection{Transmitted waves at the junctions}

By substituting Eq. (17) into the equation of motion for bending waves (1) and by considering only wave propagation in the direction of the positive $x$-axis, the transverse plate displacement is given by

$$
w_{y p}^{(j)}(x, z)=\left(A_{p}^{(j)} e^{-j k_{\mathrm{B} p x 1}^{(j)} x}+B_{p}^{(j)} e^{-j k_{\mathrm{B} p x 2}^{(j)} x}\right) e^{-j k_{\mathrm{i}} z \sin \theta_{\mathrm{i}}}
$$

for each plate $p=1 \ldots N^{(j)}$ at junction $j=1,2 . A_{p}^{(j)}$ and $B_{p}^{(j)}$ represent the complex wave amplitudes characterizing the bending waves generated on the plate. The wavenumbers $k_{\mathrm{B} p x 1}^{(j)}$ and $k_{\mathrm{B} p x 2}^{(j)}$ are given by:

$$
\begin{aligned}
& k_{\mathrm{B} p x 1}^{(j)}= \begin{cases}\sqrt{\left(k_{\mathrm{B} p}^{(j)}\right)^{2}-\left(k_{\mathrm{i}} \sin \theta_{\mathrm{i}}\right)^{2}}, & \text { if } \theta_{\mathrm{i}} \leq \theta_{\mathrm{co}} \\
-j \sqrt{\left(k_{\mathrm{i}} \sin \theta_{\mathrm{i}}\right)^{2}-\left(k_{\mathrm{B} p}^{(j)}\right)^{2}}, & \text { if } \theta_{\mathrm{i}}>\theta_{\mathrm{co}}\end{cases} \\
& k_{\mathrm{B} p x 2}^{(j)}=-j \sqrt{\left(k_{\mathrm{B} p}^{(j)}\right)^{2}+\left(k_{\mathrm{i}} \sin \theta_{\mathrm{i}}\right)^{2}} .
\end{aligned}
$$


The wavenumber $k_{\mathrm{B} p x 2}^{(j)}$ is always imaginary. The wavenumber $k_{\mathrm{B} p x 1}^{(j)}$ can be either real or imaginary, corresponding to a traveling wave or exponentially decaying near-field respectively. The angle of incidence at which the bending wave transforms from a traveling wave into a near-field is equal to the cut-off angle $\theta_{\text {co }}$ as defined in Eq. (18).

Substitution of Eq. (17) into the equations of motion (9) and (10) for quasi-longitudinal and in-plane transverse waves, leads to the following solutions:

$$
\begin{aligned}
& \Phi_{p}^{(j)}(x, z)=P_{p}^{(j)} e^{-j k_{\mathrm{L} p x}^{(j)} x} e^{-j k_{\mathrm{i}} z \sin \theta_{\mathrm{i}}}, \\
& \Psi_{p}^{(j)}(x, z)=Q_{p}^{(j)} e^{-j k_{\mathrm{T} p x}^{(j)} x} e^{-j k_{\mathrm{i}} z \sin \theta_{\mathrm{i}}},
\end{aligned}
$$

where $P_{p}^{(j)}$ and $Q_{p}^{(j)}$ are the complex amplitudes of quasi-longitudinal and in-plane transverse waves. The wavenumbers $k_{\mathrm{L} p x}^{(j)}$ and $k_{\mathrm{T} p x}^{(j)}$ are calculated as

$$
\begin{aligned}
& k_{\mathrm{L} p x}^{(j)}= \begin{cases}\sqrt{\left(k_{\mathrm{L} p}^{(j)}\right)^{2}-\left(k_{\mathrm{i}} \sin \theta_{\mathrm{i}}\right)^{2}}, & \text { if } \theta_{\mathrm{i}} \leq \theta_{\mathrm{co}} \\
-j \sqrt{\left(k_{\mathrm{i}} \sin \theta_{\mathrm{i}}\right)^{2}-\left(k_{\mathrm{L} p}^{(j)}\right)^{2}}, & \text { if } \theta_{\mathrm{i}}>\theta_{\mathrm{co}}\end{cases} \\
& k_{\mathrm{T} p x}^{(j)}= \begin{cases}\sqrt{\left(k_{\mathrm{T} p}^{(j)}\right)^{2}-\left(k_{\mathrm{i}} \sin \theta_{\mathrm{i}}\right)^{2}}, & \text { if } \theta_{\mathrm{i}} \leq \theta_{\mathrm{co}} \\
-j \sqrt{\left(k_{\mathrm{i}} \sin \theta_{\mathrm{i}}\right)^{2}-\left(k_{\mathrm{T} p}^{(j)}\right)^{2}}, & \text { if } \theta_{\mathrm{i}}>\theta_{\mathrm{co}}\end{cases}
\end{aligned}
$$

By substitution of equation (19) into equations (4)-(6), the forces and displacements corresponding to bending wave motion can be written as:

$$
\left[\begin{array}{c}
w_{y p}^{(j)} \\
\alpha_{z p}^{(j)} \\
M_{z p}^{(j)} \\
F_{y p}^{(j)}
\end{array}\right]=\left[\begin{array}{ll}
C_{p 1}^{w_{y}(j)} & C_{p 2}^{w_{y}(j)} \\
C_{p 1}^{\alpha_{z}(j)} & C_{p 2}^{\alpha_{z}(j)} \\
C_{p 1}^{M_{z}(j)} & C_{p 2}^{M_{z}(j)} \\
C_{p 1}^{F_{y}(j)} & C_{p 2}^{F_{y}(j)}
\end{array}\right]\left[\begin{array}{l}
A_{p}^{(j)} e^{-j k_{\mathrm{B} p x 1}^{(j)} x} \\
B_{p}^{(j)} e^{-j k_{\mathrm{B} p x 2}^{(j)} x}
\end{array}\right] e^{-j k_{\mathrm{i}} z \sin \theta_{\mathrm{i}}},
$$

with:

$$
\begin{aligned}
& C_{p 1}^{w_{y}(j)}=1, \\
& C_{p 2}^{w_{y}(j)}=1, \\
& C_{p 1}^{\alpha_{z}(j)}=-j k_{\mathrm{B} p x 1}^{(j)}, \\
& C_{p 2}^{\alpha_{z}(j)}=-j k_{\mathrm{B} p x 1}^{(j)}, \\
& C_{p 1}^{M_{z}(j)}=B_{p}^{\prime(j)}\left(\left(k_{\mathrm{B} p x 1}^{(j)}\right)^{2}+\nu_{p}^{(j)} k_{\mathrm{i}}^{2} \sin ^{2} \theta_{\mathrm{i}}\right), \\
& C_{p 2}^{M_{z}(j)}=B_{p}^{\prime(j)}\left(\left(k_{\mathrm{B} p x 2}^{(j)}\right)^{2}+\nu_{p}^{(j)} k_{\mathrm{i}}^{2} \sin ^{2} \theta_{\mathrm{i}}\right), \\
& C_{p 1}^{F_{y}(j)}=-j B_{p}^{\prime(j)} k_{\mathrm{B} p x 1}^{(j)}\left(\left(k_{\mathrm{B} p x 1}^{(j)}\right)^{2}+\left(2-\nu_{p}^{(j)}\right) k_{\mathrm{i}}^{2} \sin ^{2} \theta_{\mathrm{i}}\right), \\
& C_{p 2}^{F_{y}(j)}=-j B_{p}^{\prime(j)} k_{\mathrm{B} p x 2}^{(j)}\left(\left(k_{\mathrm{B} p x 2}^{(j)}\right)^{2}+\left(2-\nu_{p}^{(j)}\right) k_{\mathrm{i}}^{2} \sin ^{2} \theta_{\mathrm{i}}\right) .
\end{aligned}
$$


Similarly, the forces and displacements corresponding to in-plane wave motion can be written in terms of the contribution factors $P_{p}^{(j)}$ and $Q_{p}^{(j)}$,

$$
\left[\begin{array}{l}
w_{x p}^{(j)} \\
w_{z p}^{(j)} \\
F_{x p}^{(j)} \\
F_{z p}^{(j)}
\end{array}\right]=\left[\begin{array}{ll}
C_{p 1}^{w_{x}(j)} & C_{p 2}^{w_{x}(j)} \\
C_{p 1}^{w_{z}(j)} & C_{p 2}^{w_{z}(j)} \\
C_{p 1}^{F_{x}(j)} & C_{p 2}^{F_{x}(j)} \\
C_{p 1}^{F_{z}(j)} & C_{p 2}^{F_{z}(j)}
\end{array}\right]\left[\begin{array}{l}
P_{p}^{(j)} e^{-j k_{\mathrm{L} p x}^{(j)} x} \\
Q_{p}^{(j)} e^{-j k_{\mathrm{T} p x}^{(j)} x}
\end{array}\right] e^{-j k_{\mathrm{i}} z \sin \theta_{\mathrm{i}}},
$$

with:

$$
\begin{aligned}
& C_{p 1}^{w_{x}(j)}=-j k_{\mathrm{L} p x}^{(j)}, \\
& C_{p 2}^{w_{x}(j)}=j k_{\mathrm{i}} \sin \theta_{\mathrm{i}}, \\
& C_{p 1}^{w_{z}(j)}=-j k_{\mathrm{i}} \sin \theta_{\mathrm{i}}, \\
& C_{p 2}^{w_{z}(j)}=-j k_{\mathrm{T} p x}^{(j)}, \\
& C_{p 1}^{F_{x}(j)}=-\frac{\underline{E}_{p}^{(j)} h_{p}^{(j)}}{1-\left(\nu_{p}^{(j)}\right)^{2}}\left(\left(k_{\mathrm{L} p x}^{(j)}\right)^{2}+\nu_{p}^{(j)} k_{\mathrm{i}}^{2} \sin ^{2} \theta_{\mathrm{i}}\right), \\
& C_{p 2}^{F_{x}(j)}=2 \underline{G}_{p}^{(j)} h_{p}^{(j)} k_{\mathrm{T} p x}^{(j)} k_{\mathrm{i}} \sin \theta_{\mathrm{i}}, \\
& C_{p 1}^{F_{z}(j)}=-2 \underline{G}_{p}^{(j)} h_{p}^{(j)} k_{\mathrm{L} p x}^{(j)} k_{\mathrm{i}} \sin \theta_{\mathrm{i}}, \\
& C_{p 2}^{F_{z}(j)}=\underline{G}_{p}^{(j)} h_{p}^{(j)}\left(-\left(k_{\mathrm{T} p x}^{(j)}\right)^{2}+k_{\mathrm{i}}^{2} \sin ^{2} \theta_{\mathrm{i}}\right) .
\end{aligned}
$$

\subsubsection{Waves in the plate connecting the junction beams}

Due to the finite length of the coupling plate, there will be waves travelling in both a positive and negative direction. Following general solution is used to describe the bending wave motion in the plate connecting the two junction beams:

$$
\begin{aligned}
w_{y}^{(12)}(x, z)= & \left(A^{(12)} e^{-j k_{\mathrm{B} x 1}^{(12)}\left(x-f_{A} d\right)}+B^{(12)} e^{-j k_{\mathrm{B} x 2}^{(12)}\left(x-f_{B} d\right)}\right. \\
& \left.+C^{(12)} e^{j k_{\mathrm{B} x 1}^{(12)}\left(x-f_{C} d\right)}+D^{(12)} e^{j k_{\mathrm{B} x 2}^{(12)}\left(x-f_{D} d\right)}\right) e^{-j k_{\mathrm{i}} z \sin \theta_{\mathrm{i}}}
\end{aligned}
$$

To improve the numerical condition of the model, the wave functions are scaled so that they have amplitudes not larger than 1 in the interval $[0, d]$ with $d$ the length of the plate connecting the two junction beams [30]. 
The scaling factors are defined as

$$
\begin{aligned}
& f_{A}= \begin{cases}1, & \text { if } \Im\left\{k_{\mathrm{B} x 1}^{(12)}\right\}>0 \\
0, & \text { if } \Im\left\{k_{\mathrm{B} x 1}^{(12)}\right\} \leq 0\end{cases} \\
& f_{B}= \begin{cases}1, & \text { if } \Im\left\{k_{\mathrm{B} x 2}^{(12)}\right\}>0 \\
0, & \text { if } \Im\left\{k_{\mathrm{B} x 2}^{(12)}\right\} \leq 0\end{cases} \\
& f_{C}= \begin{cases}1, & \text { if } \Im\left\{k_{\mathrm{B} x 1}^{(12)}\right\} \leq 0 \\
0, & \text { if } \Im\left\{k_{\mathrm{B} x 1}^{(12)}\right\}>0\end{cases} \\
& f_{D}= \begin{cases}1, & \text { if } \Im\left\{k_{\mathrm{B} x 2}^{(12)}\right\} \leq 0 \\
0, & \text { if } \Im\left\{k_{\mathrm{B} x 2}^{(12)}\right\}>0\end{cases}
\end{aligned}
$$

where $\Im$ means 'imaginary part of'. The displacements and forces corresponding to bending wave motion are then given by:

$$
\left[\begin{array}{c}
w_{y}^{(12)} \\
\alpha_{z}^{(12)} \\
M_{z}^{(12)} \\
F_{y}^{(12)}
\end{array}\right]=\left[\begin{array}{cccc}
C_{1}^{w_{y}(12)} & C_{2}^{w_{y}(12)} & C_{1}^{w_{y}(12)} & C_{2}^{w_{y}(12)} \\
C_{1}^{\alpha_{z}(12)} & C_{2}^{\alpha_{z}(12)} & -C_{1}^{\alpha_{z}(12)} & -C_{2}^{\alpha_{z}(12)} \\
C_{1}^{M_{z}(12)} & C_{2}^{M_{z}(12)} & C_{1}^{M_{z}(12)} & C_{2}^{M_{z}(12)} \\
C_{1}^{F_{y}(12)} & C_{2}^{F_{y}(12)} & -C_{1}^{F_{y}(12)} & -C_{2}^{F_{y}(12)}
\end{array}\right]\left[\begin{array}{c}
A^{(12)} e^{-j k_{\mathrm{B} x 1}^{(12)}\left(x-f_{A} d\right)} \\
B^{(12)} e^{-j k_{\mathrm{B} x 2}^{(12)}\left(x-f_{B} d\right)} \\
C^{(12)} e^{j k_{\mathrm{B} x 1}^{(12)}\left(x-f_{C} d\right)} \\
D^{(12)} e^{j k_{\mathrm{B} x 2}^{(12)}\left(x-f_{D} d\right)}
\end{array}\right] e^{-j k_{\mathrm{i}} z \sin \theta_{\mathrm{i}}},
$$

where the coefficients $C$ can be calculated from equations (27)-(34). Similarly, following solutions are used to describe the in-plane wave motion,

$$
\begin{aligned}
& \Phi^{(12)}(x, z)=\left(P^{(12)} e^{-j k_{\mathrm{L} x}^{(12)}\left(x-f_{P} d\right)}+R^{(12)} e^{j k_{\mathrm{L} x}^{(12)}\left(x-f_{R} d\right)}\right) e^{-j k_{\mathrm{i}} z \sin \theta_{\mathrm{i}}}, \\
& \Psi^{(12)}(x, z)=\left(Q^{(12)} e^{-j k_{\mathrm{T} x}^{(12)}\left(x-f_{Q} d\right)}+S^{(12)} e^{j k_{\mathrm{T} x}^{(12)}\left(x-f_{S} d\right)}\right) e^{-j k_{\mathrm{i}} z \sin \theta_{\mathrm{i}}}
\end{aligned}
$$

where the scaling factors are defined as

$$
\begin{aligned}
& f_{P}= \begin{cases}1, & \text { if } \Im\left\{k_{\mathrm{L} x}^{(12)}\right\}>0 \\
0, & \text { if } \Im\left\{k_{\mathrm{L} x}^{(12)}\right\} \leq 0\end{cases} \\
& f_{Q}= \begin{cases}1, & \text { if } \Im\left\{k_{\mathrm{T} x}^{(12)}\right\}>0 \\
0, & \text { if } \Im\left\{k_{\mathrm{T} x}^{(12)}\right\} \leq 0\end{cases} \\
& f_{R}= \begin{cases}1, & \text { if } \Im\left\{k_{\mathrm{L} x}^{(12)}\right\} \leq 0 \\
0, & \text { if } \Im\left\{k_{\mathrm{L} x}^{(12)}\right\}>0\end{cases} \\
& f_{S}= \begin{cases}1, & \text { if } \Im\left\{k_{\mathrm{T} x}^{(12)}\right\} \leq 0 \\
0, & \text { if } \Im\left\{k_{\mathrm{T} x}^{(12)}\right\}>0\end{cases}
\end{aligned}
$$


These solutions lead to following relations for the in-plane displacements and forces in the coupling plate:

$$
\left[\begin{array}{c}
w_{x}^{(12)} \\
w_{z}^{(12)} \\
F_{x}^{(12)} \\
F_{z}^{(12)}
\end{array}\right]=\left[\begin{array}{cccc}
C_{1}^{w_{x}(12)} & C_{2}^{w_{x}(12)} & -C_{1}^{w_{x}(12)} & C_{2}^{w_{x}(12)} \\
C_{1}^{w_{z}(12)} & C_{2}^{w_{z}(12)} & C_{1}^{w_{z}(12)} & -C_{2}^{w_{z}(12)} \\
C_{1}^{F_{x}(12)} & C_{2}^{F_{x}(12)} & C_{1}^{F_{x}(12)} & -C_{2}^{F_{x}(12)} \\
C_{1}^{F_{z}(12)} & C_{2}^{F_{z}(12)} & -C_{1}^{F_{z}(12)} & C_{2}^{F_{z}(12)}
\end{array}\right]\left[\begin{array}{c}
P^{(12)} e^{-j k_{\mathrm{L} x}^{(12)}\left(x-f_{P} d\right)} \\
Q^{(12)} e^{-j k_{\mathrm{T} x}^{(12)}\left(x-f_{Q} d\right)} \\
R^{(12)} e^{j k_{\mathrm{L} x}^{(12)}\left(x-f_{R} d\right)} \\
S^{(12)} e^{j k_{\mathrm{T} x}^{(12)}\left(x-f_{S} d\right)}
\end{array}\right] e^{-j k_{\mathrm{i}} z \sin \theta_{\mathrm{i}}}
$$

with the coefficients $C$ given by equations (36)-(43).

\subsubsection{Incident waves}

For an incident bending wave on plate $p=1$ of junction $j=1$ that is described by the general form in Eq. (15) and impinges upon the junction at $x=0$, the displacement, rotation, bending moment and shear force are given by

$$
\begin{aligned}
w_{y 1 \mathrm{i}}^{(1)}(0, z) & =e^{-j k_{\mathrm{i}} z \sin \theta_{\mathrm{i}}} \\
\alpha_{z 1 \mathrm{i}}^{(1)}(0, z) & =j k_{\mathrm{i}} \cos \theta_{\mathrm{i}} e^{-j k_{\mathrm{i}} z \sin \theta_{\mathrm{i}}} \\
M_{z 1 \mathrm{i}}^{(1)}(0, z) & =B_{1}^{(1)} k_{\mathrm{i}}^{2}\left(\cos ^{2} \theta_{\mathrm{i}}+\nu_{1}^{(1)} \sin ^{2} \theta_{\mathrm{i}}\right) e^{-j k_{\mathrm{i}} z \sin \theta_{\mathrm{i}}}, \\
F_{y 1 \mathrm{i}}^{(1)}(0, z) & =j B_{1}^{(1)} k_{\mathrm{i}}^{3} \cos \theta_{\mathrm{i}}\left(\cos ^{2} \theta_{\mathrm{i}}+\left(2-\nu_{1}^{(1)}\right) \sin ^{2} \theta_{\mathrm{i}}\right) e^{-j k_{\mathrm{i}} z \sin \theta_{\mathrm{i}}} .
\end{aligned}
$$

where the wavenumber of the incident wave, $k_{\mathrm{i}}$, is equal to the bending wavenumber on plate 1 of junction $1, k_{\mathrm{B} 1}^{(1)}$. Since bending waves do not contribute directly to the in-plane forces and displacements, $w_{x 1 \mathrm{i}}^{(1)}, w_{z 1 \mathrm{i}}^{(1)}$, $F_{x 1 \mathrm{i}}^{(1)}$, and $F_{z 1 \mathrm{i}}^{(1)}$ are equal to zero for an incident bending wave.

A similar procedure can be applied for incident quasi-longitudinal and in-plane transverse waves. In both cases, $w_{y 1 \mathrm{i}}^{(1)}, \alpha_{z 1 \mathrm{i}}^{(1)}, M_{z 1 \mathrm{i}}^{(1)}$, and $F_{y 1 \mathrm{i}}^{(1)}$ are zero. A unit amplitude quasi-longitudinal wave that is described by the general form of Eq. (15) is given by

$$
\Phi(x, z)=e^{j k_{\mathrm{i}} x \cos \theta_{\mathrm{i}}} e^{-j k_{\mathrm{i}} z \sin \theta_{\mathrm{i}}},
$$

where $k_{\mathrm{i}}$ is equal to $k_{\mathrm{L} 1}^{(1)}$. When this wave impinges upon the first junction at $x=0$, the displacements and forces are given by

$$
\begin{aligned}
& w_{x 1 \mathrm{i}}^{(1)}(0, z)=j k_{\mathrm{i}} \cos \theta_{\mathrm{i}} e^{-j k_{\mathrm{i}} z \sin \theta_{\mathrm{i}}} \\
& w_{z 1 \mathrm{i}}^{(1)}(0, z)=-j k_{\mathrm{i}} \sin \theta_{\mathrm{i}} e^{-j k_{\mathrm{i}} z \sin \theta_{\mathrm{i}}} \\
& F_{x 1 \mathrm{i}}^{(1)}(0, z)=-\frac{\underline{E}_{1}^{(1)} h_{1}^{(1)}}{1-\left(\nu_{1}^{(1)}\right)^{2}} k_{\mathrm{i}}^{2}\left(\cos ^{2} \theta_{\mathrm{i}}+\nu_{1}^{(1)} \sin ^{2} \theta_{\mathrm{i}}\right) e^{-j k_{\mathrm{i}} z \sin \theta_{\mathrm{i}}}, \\
& F_{y 1 \mathrm{i}}^{(1)}(0, z)=2 G_{1}^{(1)} h_{1}^{(1)} k_{\mathrm{i}}^{2} \cos \theta_{\mathrm{i}} \sin \theta_{\mathrm{i}} e^{-j k_{\mathrm{i}} z \sin \theta_{\mathrm{i}}} .
\end{aligned}
$$

Similarly, a unit amplitude transverse wave is given by

$$
\Psi(x, z)=e^{j k_{\mathrm{i}} x \cos \theta_{\mathrm{i}}} e^{-j k_{\mathrm{i}} z \sin \theta_{\mathrm{i}}},
$$


where $k_{\mathrm{i}}$ is equal to $k_{\mathrm{T} 1}^{(1)}$. When this wave impinges upon the first junction at $x=0$, the in-plane displacements and forces are given by

$$
\begin{aligned}
& w_{x 1 \mathrm{i}}^{(1)}(0, z)=j k_{\mathrm{i}} \sin \theta_{\mathrm{i}} e^{-j k_{\mathrm{i}} z \sin \theta_{\mathrm{i}}}, \\
& w_{z 1 \mathrm{i}}^{(1)}(0, z)=j k_{\mathrm{i}} \cos \theta_{\mathrm{i}} e^{-j k_{\mathrm{i}} z \sin \theta_{\mathrm{i}}} \\
& F_{x 1 \mathrm{i}}^{(1)}(0, z)=-2 G_{1}^{(1)} h_{1}^{(1)} k_{\mathrm{i}}^{2} \cos \theta_{\mathrm{i}} \sin \theta_{\mathrm{i}} e^{-j k_{\mathrm{i}} z \sin \theta_{\mathrm{i}}}, \\
& F_{y 1 \mathrm{i}}^{(1)}(0, z)=G_{1}^{(1)} h_{1}^{(1)} k_{\mathrm{i}}^{2}\left(\sin ^{2} \theta_{\mathrm{i}}-\cos ^{2} \theta_{\mathrm{i}}\right) e^{-j k_{\mathrm{i}} z \sin \theta_{\mathrm{i}}} .
\end{aligned}
$$

\subsection{Boundary and continuity conditions}

Because the solution functions are exact solutions of the equations of motion, the complex amplitudes are only determined by the boundary and continuity conditions at the rigid junction beams. The equilibrium conditions of the junction beams are expressed in terms of the global coordinate system (figure 3 ). For the first junction beam, this yields:

$$
\begin{aligned}
& \sum_{p=1}^{N^{(1)}}\left[F_{x p}^{(1)}(0, z) \cos \theta_{p}^{(1)}-F_{y p}^{(1)}(0, z) \sin \theta_{p}^{(1)}\right]+F_{x}^{(12)}(0, z) \cos \theta^{(12)}-F_{y}^{(12)}(0, z) \sin \theta^{(12)} \\
& \quad+F_{x 1 i}^{(1)}(0, z) \cos \theta_{1}^{(1)}-F_{y 1 i}^{(1)}(0, z) \sin \theta_{1}^{(1)}=0, \\
& \sum_{p=1}^{N^{(1)}}\left[F_{x p}^{(1)}(0, z) \sin \theta_{p}^{(1)}+F_{y p}^{(1)}(0, z) \cos \theta_{p}^{(1)}\right]+F_{x}^{(12)}(0, z) \sin \theta^{(12)}+F_{y}^{(12)}(0, z) \cos \theta^{(12)} \\
& \quad+F_{x 1 \mathrm{i}}^{(1)}(0, z) \sin \theta_{1}^{(1)}+F_{y 1 \mathrm{i}}^{(1)}(0, z) \cos \theta_{1}^{(1)}=0, \\
& \sum_{p=1}^{N^{(1)}}\left[F_{z p}^{(1)}(0, z)\right]+F_{z}^{(12)}(0, z)+F_{z 1 \mathrm{i}}^{(1)}(0, z)=0, \\
& \sum_{p=1}^{N^{(1)}}\left[M_{z p}^{(1)}(0, z)\right]+M_{z}^{(12)}(0, z)+M_{z 1 \mathrm{i}}^{(1)}(0, z)=0 .
\end{aligned}
$$

Similarly, the equilibrium conditions of the second junction beam are given by:

$$
\begin{aligned}
& \sum_{p=1}^{N^{(2)}}\left[F_{x p}^{(2)}(0, z) \cos \theta_{p}^{(2)}-F_{y p}^{(2)}(0, z) \sin \theta_{p}^{(2)}\right]-F_{x}^{(12)}(d, z) \cos \theta^{(12)}+F_{y}^{(12)}(d, z) \sin \theta^{(12)}=0, \\
& \sum_{p=1}^{N^{(2)}}\left[F_{x p}^{(2)}(0, z) \sin \theta_{p}^{(2)}+F_{y p}^{(2)}(0, z) \cos \theta_{p}^{(2)}\right]-F_{x}^{(12)}(0, z) \sin \theta^{(12)}-F_{y}^{(12)}(d, z) \cos \theta^{(12)}=0, \\
& \sum_{p=1}^{N^{(2)}}\left[F_{z p}^{(2)}(0, z)\right]-F_{z}^{(12)}(d, z)=0, \\
& \sum_{p=1}^{N^{(2)}}\left[M_{z p}^{(2)}(0, z)\right]-M_{z}^{(12)}(d, z)=0 .
\end{aligned}
$$


Here, $\theta_{p}^{(j)}$ is the coupling angle (figure 3 ) and $N^{(j)}$ is the number of plates connected to junction $j$ without the coupling plate. These equilibrium conditions ensure that the sum of forces acting on each junction beam equals zero.

For a rigid plate junction, the displacements at the plate edge $\left(w_{x p}^{(j)}, w_{y p}^{(j)}, w_{z p}^{(j)}\right.$, and $\left.\alpha_{z p}^{(j)}\right)$ are assumed to be equal to the displacements of the junction beam $\left(w_{x \mathrm{~b}}^{(j)}, w_{y \mathrm{~b}}^{(j)}, w_{z \mathrm{~b}}^{(j)}\right.$, and $\left.\alpha_{z \mathrm{~b}}^{(j)}\right)$. The continuity conditions at the junction beam are written in the local coordinate system:

$$
\begin{aligned}
& w_{x p}^{(j)}(0, z)=w_{x \mathrm{~b}}^{(j)}(z) \cos \theta_{p}^{(j)}+w_{y \mathrm{~b}}^{(j)}(z) \sin \theta_{p}^{(j)}, \\
& w_{y p}^{(j)}(0, z)=-w_{x \mathrm{~b}}^{(j)}(z) \sin \theta_{p}^{(j)}+w_{y \mathrm{~b}}^{(j)}(z) \cos \theta_{p}^{(j)}, \\
& w_{z p}^{(j)}(0, z)=w_{z \mathrm{~b}}^{(j)}(z), \\
& \alpha_{z p}^{(j)}(0, z)=\alpha_{z \mathrm{~b}}^{(j)}(z) .
\end{aligned}
$$

For the specific case of the first plate $(p=1, j=1)$, the contributions of the incident wave $\left(w_{x 1 \mathrm{i}}^{(1)}, w_{y 1 \mathrm{i}}^{(1)}, w_{z 1 \mathrm{i}}^{(1)}\right.$, and $\left.\alpha_{z 1 \mathrm{i}}^{(1)}\right)$ to the displacements at the plate edge have to be added to the left-hand side of the corresponding equations:

$$
\begin{aligned}
& w_{x 1}^{(1)}(0, z)+w_{x 1 \mathrm{i}}^{(1)}(z)=w_{x \mathrm{~b}}^{(1)}(z) \cos \theta_{1}^{(1)}+w_{y \mathrm{~b}}^{(1)}(z) \sin \theta_{1}^{(1)}, \\
& w_{y 1}^{(1)}(0, z)+w_{y 1 \mathrm{i}}^{(1)}(z)=-w_{x \mathrm{~b}}^{(1)}(z) \sin \theta_{1}^{(1)}+w_{y \mathrm{~b}}^{(1)}(z) \cos \theta_{1}^{(1)}, \\
& w_{z 1}^{(1)}(0, z)+w_{z 1 \mathrm{i}}^{(1)}(z)=w_{z \mathrm{~b}}^{(1)}(z), \\
& \alpha_{z 1}^{(1)}(0, z)+\alpha_{z 1 \mathrm{i}}^{(1)}(z)=\alpha_{z \mathrm{~b}}^{(1)}(z) .
\end{aligned}
$$

For the plate connecting the two junction beams, following continuity conditions hold:

$$
\begin{aligned}
& w_{x}^{(12)}(0, z)=w_{x \mathrm{~b}}^{(1)}(z) \cos \theta^{(12)}+w_{y \mathrm{~b}}^{(1)}(z) \sin \theta^{(12)}, \\
& w_{y}^{(12)}(0, z)=-w_{x \mathrm{~b}}^{(1)}(z) \sin \theta^{(12)}+w_{y \mathrm{~b}}^{(1)}(z) \cos \theta^{(12)}, \\
& w_{z}^{(12)}(0, z)=w_{z \mathrm{~b}}^{(1)}(z), \\
& \alpha_{z}^{(12)}(0, z)=\alpha_{z \mathrm{~b}}^{(1)}(z), \\
& w_{x}^{(12)}(d, z)=w_{x \mathrm{~b}}^{(2)}(z) \cos \theta^{(12)}+w_{y \mathrm{~b}}^{(2)}(z) \sin \theta^{(12)}, \\
& w_{y}^{(12)}(d, z)=-w_{x \mathrm{~b}}^{(2)}(z) \sin \theta^{(12)}+w_{y \mathrm{~b}}^{(2)}(z) \cos \theta^{(12)}, \\
& w_{z}^{(12)}(d, z)=w_{z \mathrm{~b}}^{(2)}(z) \\
& \alpha_{z}^{(12)}(d, z)=\alpha_{z \mathrm{~b}}^{(2)}(z) .
\end{aligned}
$$

The set of equations (71)-(94) are solved by converting them into matrix format and using a matrix inversion to give the complex amplitudes on each plate $\left(A_{p}^{(j)}, B_{p}^{(j)}, Q_{p}^{(j)}, R_{p}^{(j)}\right)$ as well as the junction beam parameters $\left(w_{x \mathrm{~b}}^{(j)}, w_{y \mathrm{~b}}^{(j)}, w_{z \mathrm{~b}}^{(j)}\right.$, and $\left.\alpha_{z \mathrm{~b}}^{(j)}\right)$. 


\subsection{Transmission coefficients}

The transmission coefficient for any incident wave on plate 1 of junction 1 that is transmitted to plate $p$ of junction $j$ at a specific angle of incidence is calculated from the ratio of the wave intensities in the $x$-direction using

$$
\tau\left(\theta_{\mathrm{i}}\right)=\frac{I_{x p}^{(j)}\left(\theta_{\mathrm{i}}\right)}{I_{x 1 \mathrm{i}}^{(1)}\left(\theta_{\mathrm{i}}\right)}
$$

For a unit amplitude wave incident upon the junction, the $x$-direction intensities are [9]

$$
\begin{aligned}
& I_{\mathrm{B} x 1 \mathrm{i}}^{(1)}\left(\theta_{\mathrm{i}}\right)=B_{1}^{(1)} \omega\left(k_{\mathrm{B} 1}^{(1)}\right)^{3} \cos \theta_{\mathrm{i}}, \\
& I_{\mathrm{L} x 1 \mathrm{i}}^{(1)}\left(\theta_{\mathrm{i}}\right)=\frac{1}{2} m_{1}^{\prime \prime(1)} \omega^{3} k_{\mathrm{L} 1}^{(1)} \cos \theta_{\mathrm{i}}, \\
& I_{\mathrm{T} x 1 \mathrm{i}}^{(1)}\left(\theta_{\mathrm{i}}\right)=\frac{1}{2} m_{1}^{\prime \prime(1)} \omega^{3} k_{\mathrm{T} 1}^{(1)} \cos \theta_{\mathrm{i}},
\end{aligned}
$$

for an incident bending, quasi-longitudinal, and in-plane transverse wave, respectively. For the transmitted bending wave on plate $p$ of junction $j$, the $x$-direction intensity is [9]

$$
I_{\mathrm{B} x p}^{(j)}\left(\theta_{\mathrm{i}}\right)=\frac{1}{2} \Re\left\{F_{y p}^{(j)}\left(-j \omega w_{y p}^{(j)}\right)^{*}+M_{z p}^{(j)}\left(j \omega \alpha_{z p}^{(j)}\right)^{*}\right\},
$$

where $*$ denotes the complex conjugate and $\Re$ means 'real part of'. The total in-plane wave intensity in the $x$-direction $I_{\mathrm{LT} p x}^{(j)}$ is composed of contributions from the quasi-longitudinal and the in-plane transverse wave, and is given by

$$
I_{\mathrm{LT} x p}^{(j)}\left(\theta_{\mathrm{i}}\right)=\frac{1}{2} \Re\left\{F_{x p}^{(j)}\left(-j \omega w_{x p}^{(j)}\right)^{*}+F_{z p}^{(j)}\left(-j \omega w_{z p}^{(j)}\right)^{*}\right\} .
$$

The transmitted wave intensities can be re-written in terms of the complex wave amplitudes [29]

$$
\begin{aligned}
& I_{\mathrm{B} x p}^{(j)}\left(\theta_{\mathrm{i}}\right)=-\frac{1}{2} \omega \Im\left\{C_{p 1}^{F_{y}(j)}\left(C_{p 1}^{w_{y}(j)}\right)^{*}-C_{p 1}^{M_{z}(j)}\left(C_{p 1}^{\alpha_{z}(j)}\right)^{*}\right\}\left|A_{p}^{(j)}\right|^{2}, \\
& I_{\mathrm{L} x p}^{(j)}\left(\theta_{\mathrm{i}}\right)=-\frac{1}{2} \omega \Im\left\{C_{p 1}^{F_{x}(j)}\left(C_{p 1}^{w_{x}(j)}\right)^{*}+C_{p 1}^{F_{z}(j)}\left(C_{p 1}^{w_{z}(j)}\right)^{*}\right\}\left|P_{p}^{(j)}\right|^{2}, \\
& I_{\mathrm{T} x p}^{(j)}\left(\theta_{\mathrm{i}}\right)=-\frac{1}{2} \omega \Im\left\{C_{p 2}^{F_{x}(j)}\left(C_{p 2}^{w_{x}(j)}\right)^{*}+C_{p 2}^{F_{z}(j)}\left(C_{p 2}^{F_{z}(j)}\right)^{*}\right\}\left|Q_{p}^{(j)}\right|^{2} .
\end{aligned}
$$

The wave approach considers a plane wave that impinges upon the junction at a specific angle of incidence $\theta_{\mathrm{i}}$. This results in an angle-specific transmission coefficient, $\tau\left(\theta_{\mathrm{i}}\right)$. In the framework of SEA, it is assumed that the incident energy is uniformly distributed in angle. In this case, one is interested in the angular average transmission coefficient, $\overline{\tau_{\mathrm{i}}}$, that is given by $[26,29]$

$$
\bar{\tau}=\int_{0}^{\pi / 2} \tau\left(\theta_{\mathrm{i}}\right) \cos \theta_{\mathrm{i}} \mathrm{d} \theta_{\mathrm{i}}
$$




\subsection{Numerical examples}

The theory discussed in this section is illustrated by a number of numerical examples. The junctions considered are composed of concrete plates with material properties $\rho=2500 \mathrm{~kg} / \mathrm{m}^{3}, E=30 e 9 \mathrm{~Pa}, \nu=0.17$, and $\eta=0$. The structure-borne sound transmission at a double wall $\mathrm{T}$-junction (figure $1 \mathrm{~b}$ ) is compared with that of a single wall T-junction (figure 1a). The floors (plates 1 and 2 in figure 1) have a thickness of $15 \mathrm{~cm}$ and the walls (plates 3 and 4 in figure 1) a thickness of $10 \mathrm{~cm}$. The cavity width $d$ of the double wall is $5 \mathrm{~cm}$. The transmission coefficients are calculated as a function of the angle of incidence for the case of an incident bending wave at $1000 \mathrm{~Hz}$. The values of the transmission coefficients are quantified by the distance between two adjacent curves in the cumulative diagram of figure 4 . The results clearly show that the sum of transmission coefficients is equal to unity for all angles of incidence as required by the conservation of energy. The cut-off angle for quasi-longitudinal waves is equal to $16.2^{\circ}$ at $1000 \mathrm{~Hz}$, above which no quasilongitudinal waves are transmitted. Similarly, the cut-off angle for transverse waves equals $25.6^{\circ}$ at $1000 \mathrm{~Hz}$. These cut-off angles can be clearly identified in the results for both the single and double wall junction. At larger angles of incidence, all of the incident intensity is reflected or transmitted as bending waves. The general trends for the single and double wall junction are similar. More wave energy is reflected by the double wall compared to the single wall. In general, the bending wave transmission to the second plate is smaller for the double wall than for the single wall junction. In the double wall results, the transmission coefficients for the fourth plate are smaller than for the third plate. While the sum of the bending wave energy transmitted to the double wall leafs is larger than for the single wall, the transmission coefficients for each leaf is smaller than for the single wall.

To investigate the influence of in-plane wave motion, a model has been set up using the wave approach assuming that there are only bending waves. For this purpose, the junction beams are simply supported so that they cannot undergo displacement, but are free to rotate. Figure 5 compares the angular average transmission loss $\mathrm{TL}=-10 \log \bar{\tau}$ obtained with the two wave approaches for the single and double wall $\mathrm{T}-$ junctions described above. For the single wall junction, the TL is independent of frequency when the wave approach assumes that there are only bending waves (figure 5a). While the bending wave only approach gives reasonable results for the single wall junction, it largely overestimates the TL for the double wall junction (figure 5b). This strong overestimation is probably caused by modeling the junctions as simply supported beams to avoid the generation of in-plane waves. Because the two junctions at close distance cannot undergo displacement, the generation of bending waves is also restricted by these additional boundary conditions, especially at low frequencies where the bending wavelength is much larger than the cavity width. These results also indicate that a significant amount of energy can be transported between the two junction beams by in-plane wave transmission as observed in previous investigations [19, 23]. The simplified bending only model is thus insufficient for an accurate prediction of the structure-borne sound transmission across double wall junctions and in-plane waves should be accounted for. 


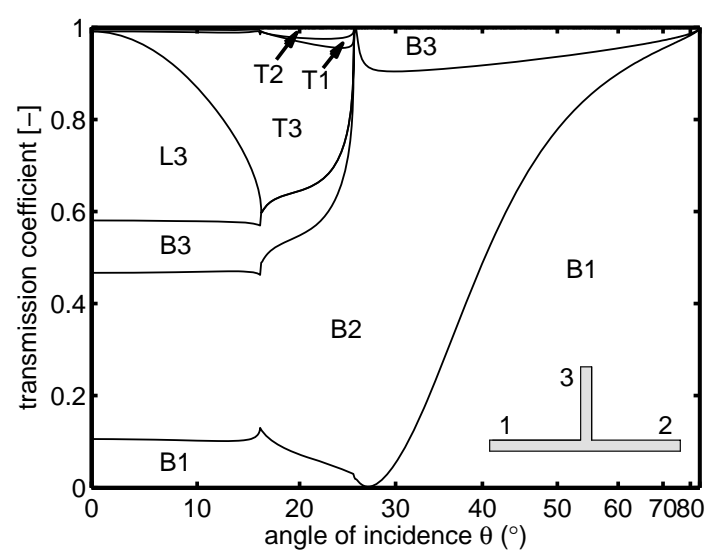

(a)

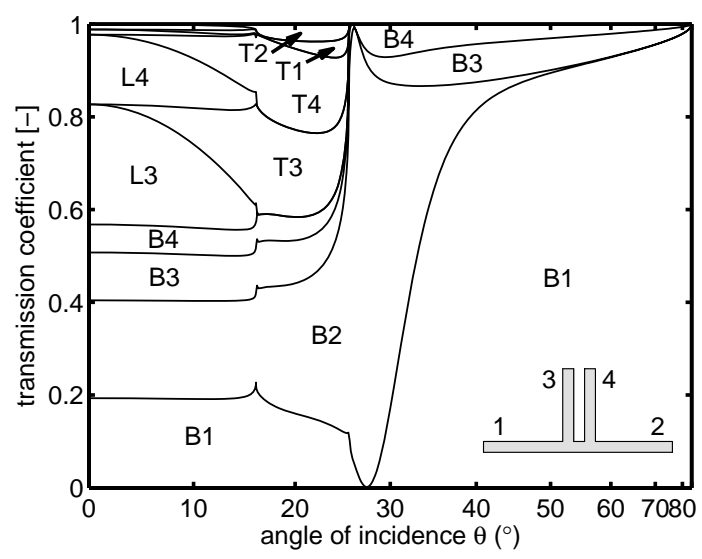

(b)

Figure 4: Cumulative diagram showing the transmission/reflection coefficient as a function of the angle of incidence for (a) a concrete single wall T-junction $\left(h_{1}=h_{2}=0.15 \mathrm{~m}, h_{3}=0.10 \mathrm{~m}\right)$ and (b) a concrete double wall T-junction $\left(h_{1}=h_{2}=0.15 \mathrm{~m}\right.$, $\left.h_{3}=h_{4}=0.10 \mathrm{~m}, d=5 \mathrm{~cm}\right)$ for an incident bending wave on plate 1 at $1000 \mathrm{~Hz}$. The descriptors $\mathrm{B}_{j} / \mathrm{L}_{j} / \mathrm{T}_{j}$ refer to a transmitted/reflected bending wave (B) / quasi-longitudinal wave (L) / in-plane transverse wave (T) on plate $j$.

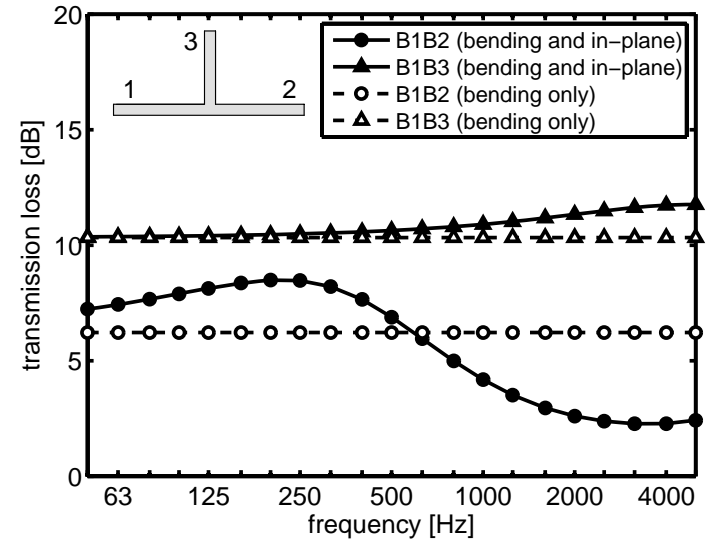

(a)

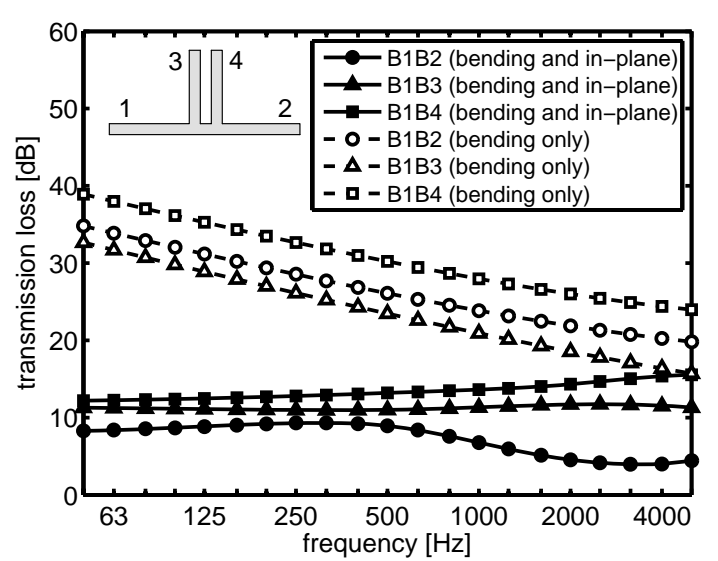

(b)

Figure 5: Angular average transmission loss for bending waves transmitted across (a) a concrete single wall T-junction $\left(h_{1}=\right.$ $\left.h_{2}=0.15 \mathrm{~m}, h_{3}=0.10 \mathrm{~m}\right)$ and (b) a concrete double wall T-junction $\left(h_{1}=h_{2}=0.15 \mathrm{~m}, h_{3}=h_{4}=0.10 \mathrm{~m}, d=5 \mathrm{~cm}\right)$ for an incident bending wave on plate 1 . The results are calculated with a wave approach for bending waves only and a wave approach for bending and in-plane waves.

\section{Statistical model}

\subsection{Statistical energy analysis (SEA)}

The angular average transmission coefficients determined from the wave theory of section 2 can be used in a SEA model [8] to predict the velocity level differences between junctions built up of finite plates. Each 
plate is represented by three subsystems to incorporate bending, quasi-longitudinal, and in-plane transverse waves into the SEA model. The plate connecting the two junction beams is not taken into account explicitly as a separate subsystem in the SEA model. The SEA model for the double plate junction thus consists of $3\left(N^{(1)}+N^{(2)}\right)$ subsystems. For isotropic, line connected plates, the coupling loss factors between the different subsystems can be determined from the angular average transmission coefficient by [29]:

$$
\eta_{i j}=\frac{c_{\mathrm{g} i} L_{i j} \bar{\tau}_{i j}}{\omega \pi S_{i}}
$$

where $L_{i j}$ is the junction length, $S_{i}$ is the surface area of plate $i$, and $c_{\mathrm{g} i}$ is the group velocity for the waves (bending, quasi-longitudinal or transverse) on plate $i$. These coupling loss factors can be used in a general SEA matrix formulation which can be found in appendix Appendix A.1. Solving the SEA equations gives the energy of each subsystem. In this paper, we are interested in the velocity level difference $D_{\mathrm{v}, i j}$ between the excited plate $i$ and plate $j$, which can be calculated from the bending energy ratio,

$$
D_{\mathrm{v}, i j}=10 \log \frac{\left|v_{y, i}\right|^{2}}{\left|v_{y, j}\right|^{2}}=10 \log \frac{E_{\mathrm{B}_{i}}}{E_{\mathrm{B}_{j}}}+10 \log \frac{m_{j}^{\prime \prime}}{m_{i}^{\prime \prime}},
$$

with $v_{y, i / j}$ the transverse velocity and $E_{\mathrm{B}_{i / j}}$ the bending energy of plate $i / j$.

\subsection{Numerical results}

The SEA model is used to investigate the vibration transmission across double wall junctions. The results are compared with results for rigid single wall junctions. In all calculations, the junction beams have a length of $4 \mathrm{~m}$, the floors (plates 1 and 2 in figure 1) have a width of $5 \mathrm{~m}$ and the walls (plates 3 to 6 in figure 1) have a height of $3.5 \mathrm{~m}$. An internal loss factor of 0.01 is used in the SEA model for the plates and no edge damping is included (apart from coupling to the other plates).

\subsubsection{Double concrete wall junctions}

First, a $\mathrm{T}$ - and $\mathrm{X}$-junction composed of $15 \mathrm{~cm}$ thick concrete floors and double concrete walls are considered. The double walls consist of $10 \mathrm{~cm}$ concrete leafs separated by an air cavity of $5 \mathrm{~cm}$. The material properties used for the concrete plates are given in table 1. Figure 6 shows the velocity level difference for the T- junction when the floor (plate 1) or the wall (plate 3) is excited. Figure 7 shows the results for the $\mathrm{X}$ - junction. The results for $D_{\mathrm{v}, 15}$ and $D_{\mathrm{v}, 16}$ are not shown because $D_{\mathrm{v}, 15}=D_{\mathrm{v}, 13}$ and $D_{\mathrm{v}, 16}=D_{\mathrm{v}, 14}$ due to symmetry.

For transmission around the corner, i.e. from floor to wall or from wall to floor, the velocity level difference steadily increases with frequency for both the T-junction (figure 6) and the $\mathrm{X}$-junction (figure 7). The velocity level difference between a floor and the nearest wall $\left(D_{\mathrm{v}, 13}\right.$ and $\left.D_{\mathrm{v}, 31}\right)$ is smaller than the velocity level difference between a floor and the furthest wall $\left(D_{\mathrm{v}, 14}\right.$ and $\left.D_{\mathrm{v}, 32}\right)$. This can be expected because a wall is added on the transmission path. The difference is however negligible at low frequencies 


\begin{tabular}{lcccc}
\hline Material & $\rho\left[\mathrm{kg} / \mathrm{m}^{3}\right]$ & $E[\mathrm{MPa}]$ & $\nu[-]$ & $\eta[-]$ \\
\hline Concrete & 2500 & 30000 & 0.17 & 0.01 \\
Cellular concrete & 650 & 1800 & 0.30 & 0.01 \\
Gypsum plasterboard & 700 & 2500 & 0.30 & 0.01 \\
\hline
\end{tabular}

Table 1: Material data (density $\rho$, Young's modulus $E$, Poisson's ratio $\nu$, and internal loss factor $\eta$ ) used in the simulations.

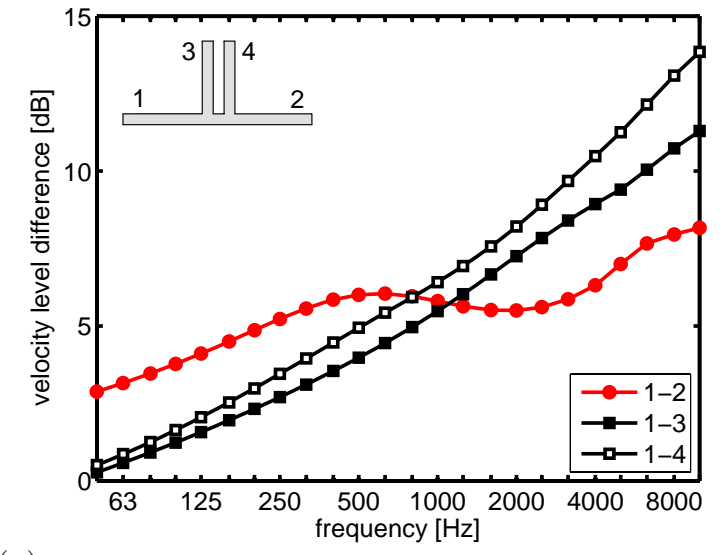

(a)

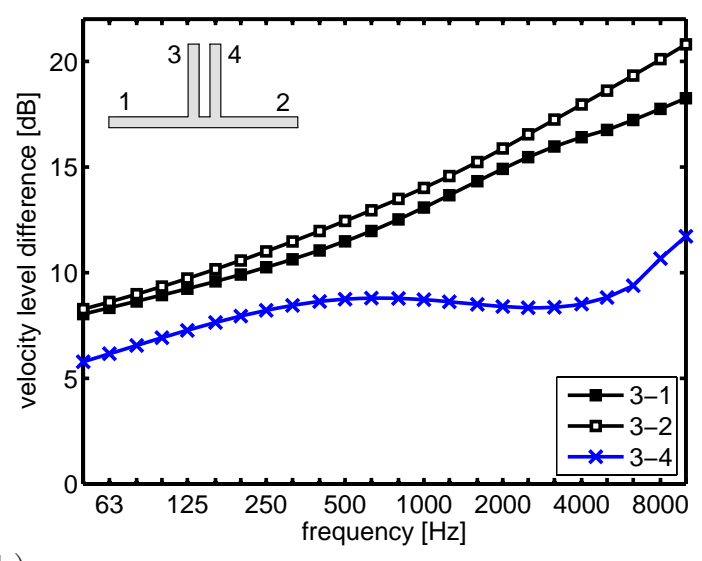

(b)

Figure 6: Velocity level difference for a concrete double wall T-junction $\left(h_{1}=h_{2}=0.15 \mathrm{~m}, h_{3}=h_{4}=0.10 \mathrm{~m}, d=5 \mathrm{~cm}\right)$ when (a) plate 1 or (b) plate 3 is excited.

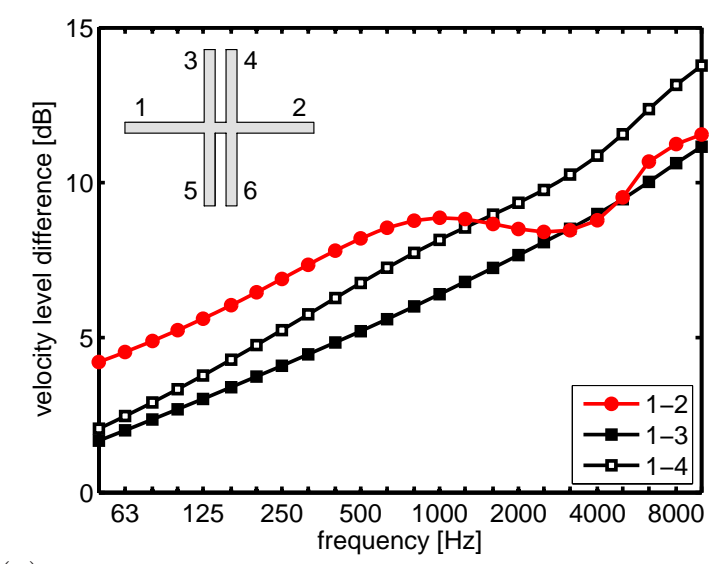

(a)

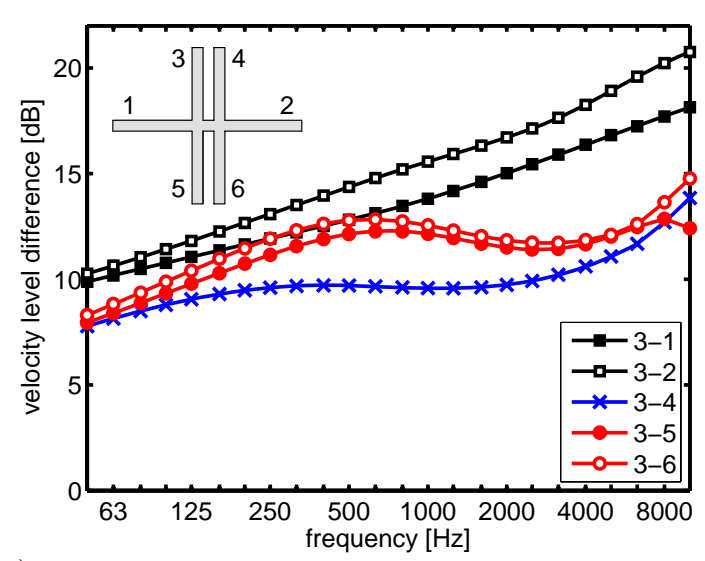

(b)

Figure 7: Velocity level difference for a concrete double wall X-junction $\left(h_{1}=h_{2}=0.15 \mathrm{~m}, h_{3}=h_{4}=h_{5}=h_{6}=0.10 \mathrm{~m}\right.$, $d=5 \mathrm{~cm}$ ) when (a) plate 1 or (b) plate 3 is excited.

and increases up to $2.5 \mathrm{~dB}$ at $10000 \mathrm{~Hz}$. The floor-wall vibration attenuation for the $\mathrm{X}$-junction is similar to that for the $\mathrm{T}$-junction, but on average $1.5 \mathrm{~dB}$ larger in the frequency range up to $2000 \mathrm{~Hz}$. At higher 
frequencies, the difference is negligible.

The floor-floor velocity level difference $D_{\mathrm{v}, 12}$ generally increases with frequency but a plateau value is observed between 500 and $4000 \mathrm{~Hz}$. The vibration reduction is again the largest for the $\mathrm{X}$-junction. The difference between the $\mathrm{X}$ - and $\mathrm{T}-\mathrm{junction}$ is $1.5 \mathrm{~dB}$ at low frequencies and $3 \mathrm{~dB}$ at high frequencies. Again, it is evident that the attenuation will increase when one or more walls are added on the transmission path.

The wall-wall velocity level difference $D_{\mathrm{v}, 34}$ is 5 to $10 \mathrm{~dB}$ for the T-junction and about $10 \mathrm{~dB}$ for the $\mathrm{X}$-junction. It must be reminded that airborne coupling between the walls is not accounted for in the model. The airborne coupling however reduces strongly above the mass-spring-mass resonance frequency of the double wall at $24 \mathrm{~Hz}$. The velocity level difference between the two walls due to airborne coupling as predicted with an infinite plate model for normal incidence, is already more than $15 \mathrm{~dB}$ at $50 \mathrm{~Hz}$. The structure-borne velocity level difference between the walls is limited to $5-10 \mathrm{~dB}$ in the entire frequency range of interest. For heavyweight structures, the airborne coupling between the walls can thus be disregarded in the entire frequency range of interest.

For the $\mathrm{X}$-junction, there are two additional flanking paths from the upper wall to the lower walls, $D_{\mathrm{v}, 35}$ and $D_{\mathrm{v}, 36}$. For both flanking paths, the velocity level difference is similar. $D_{\mathrm{v}, 35}$ and $D_{\mathrm{v}, 36}$ show the same trends as $D_{\mathrm{v}, 12}$ between the two floors, but are on average $4 \mathrm{~dB}$ larger.

It can be noted here that the SEA model is not applicable at low frequencies as it doesn't account for modal behaviour of the plates. For the concrete walls and floors, the modal overlap factor exceeds unity above $1600 \mathrm{~Hz}$ only. Large variations are therefore possible for a specific junction. However, previous investigations $[8,26,27]$ have indicated that SEA can give reasonable results even when these criteria are not met. A comparison with numerical simulations for finite-sized plates has indeed indicated that SEA can give good insight in the general trends even at low frequencies [28].

Although predictions have been shown up to $10000 \mathrm{~Hz}$, the applicability to real heavyweight building structures is limited at frequencies above $5000 \mathrm{~Hz}$. At high frequencies, the assumption of perfectly rigid coupling between the plates is often not valid anymore due to imperfect joints. Furthermore, the vibration level will decrease with distance across the plates due to high damping and thus the assumption of diffuse vibration fields is violated. This attenuation will be stronger for brick- and blockwork walls.

\subsubsection{Comparison with single wall junctions}

Figure 8 compares the velocity level difference of the double wall $\mathrm{X}$-junction of the previous paragraph with that of a single wall $\mathrm{X}$-junction when the floor is excited. The single concrete walls have a thickness of $10 \mathrm{~cm}$. The velocity level difference $D_{\mathrm{v}, 12}$ between floor and floor for the double wall shows the same trends as for the single wall junction, but the vibration reduction for the double walls is $1.5-3.5 \mathrm{~dB}$ larger over the entire frequency range of interest. Similarly, $D_{\mathrm{v}, 14}$ between the floor and the second cavity leaf is on average $3 \mathrm{~dB}$ larger than the floor-wall velocity level difference $D_{\mathrm{v}, 13}$ of the single wall junction. At high 


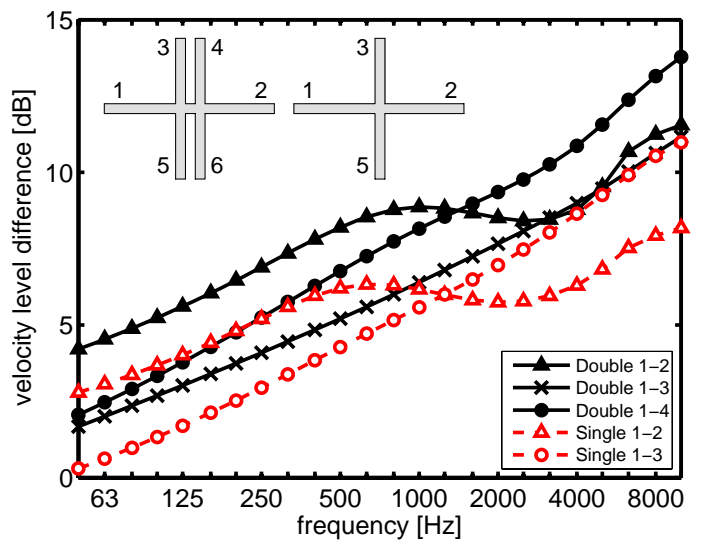

Figure 8: Velocity level difference for a X-junction with concrete floors $\left(h_{1}=h_{2}=0.15 \mathrm{~m}\right)$ and single concrete walls $\left(h_{3}=\right.$ $\left.h_{5}=0.10 \mathrm{~m}\right)$ or double concrete walls $\left(h_{3}=h_{4}=h_{5}=h_{6}=0.10 \mathrm{~m}, d=0.05 \mathrm{~m}\right)$ when plate 1 is excited.

frequencies, the velocity level difference $D_{\mathrm{v}, 13}$ for the double wall converges to the floor-wall velocity level difference $D_{\mathrm{v}, 13}$ of the single wall junction, indicating that the second leaf of the double wall is decoupled from the first wall. At low frequencies, however, both leafs of the double wall are strongly coupled. As a result, $D_{\mathrm{v}, 13}$ is similar to $D_{\mathrm{v}, 14}$ for the double wall in this frequency range, and larger than the floor-wall velocity level difference of the single wall junction.

The difference between the floor-floor transmission for the double and single concrete wall $\mathrm{X}$-junctions $\left(D_{v, 12}^{\text {double }}-D_{v, 12}^{\text {single }}\right)$ is plotted in figure 9a. Similarly, figure $9 \mathrm{~b}$ shows the difference for the floor-wall transmission $\left(D_{v, 14}^{\text {double }}-D_{v, 13}^{\text {single }}\right)$. The same comparison is made between a single and double wall T-junction composed of $10 \mathrm{~cm}$ concrete walls, and between single and double wall $\mathrm{X}$-junctions composed of $20 \mathrm{~cm}$ concrete walls, $10 \mathrm{~cm}$ cellular concrete walls, or $12.5 \mathrm{~mm}$ gypsum fiberboard walls. All junctions have $15 \mathrm{~cm}$ concrete floors. For the concrete and cellular concrete walls, the cavity width is $5 \mathrm{~cm}$. The double gypsum fiberboard walls have a cavity width of $205 \mathrm{~mm}$. The material parameters used for the different walls are summarized in table 1 . The difference between a double and a single concrete wall $\mathrm{T}$-junction with $10 \mathrm{~cm}$ concrete walls is $1-2 \mathrm{~dB}$. This difference is on average $1 \mathrm{~dB}$ smaller than for the $\mathrm{X}$-junction. When the wall thickness of the $\mathrm{X}$-junction is increased to $20 \mathrm{~cm}$, the differences between the single and double wall junctions increase to $3-5 \mathrm{~dB}$. For the lightweight cellular concrete walls, the differences are limited to $1 \mathrm{~dB}$, while the differences for the gypsum fiberboard walls are negligible. The difference $D_{v}^{\text {double }}-D_{v}^{\text {single }}$ thus increases with increasing mass of the double leaf walls and is larger for a $\mathrm{X}$-junction than for a $\mathrm{T}$-junction.

\subsubsection{Equivalent single wall modeling}

It is interesting to verify whether the vibration transmission across double wall junctions can be modelled using an equivalent single wall model. A double wall $\mathrm{T}$-junction is often modeled as a single wall $\mathrm{X}$-junction. 


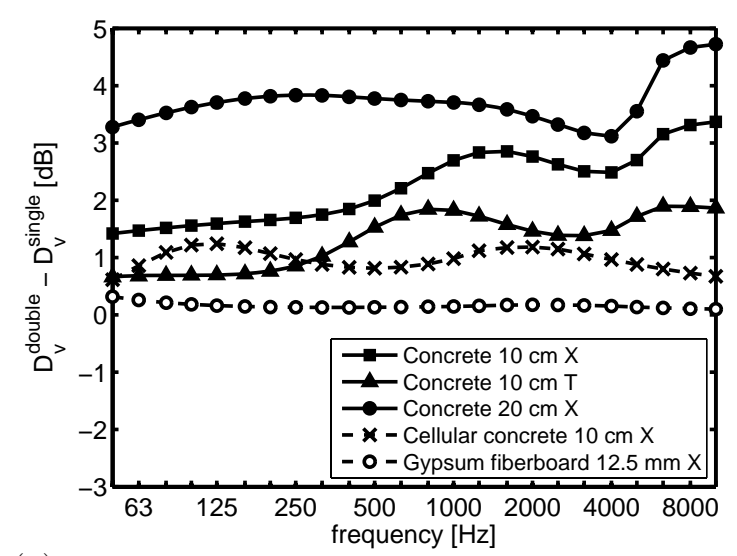

(a)

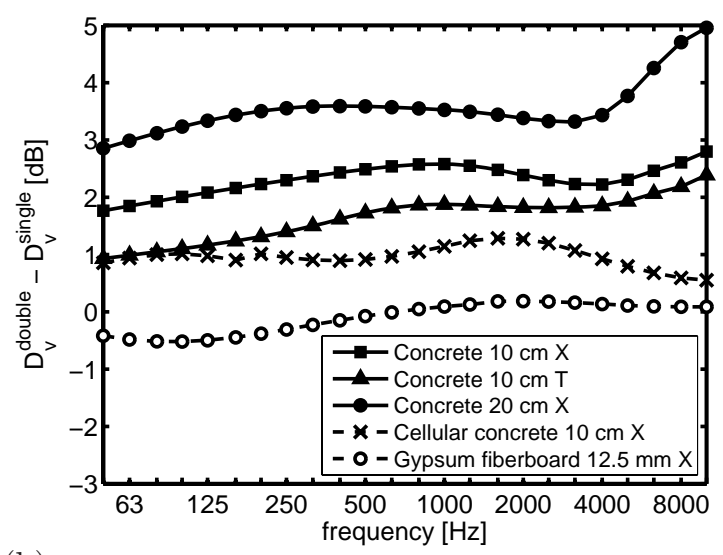

(b)

Figure 9: Difference between the velocity level difference of double wall junctions and the velocity level difference of single wall junctions for (a) the floor-floor transmission $\left(D_{v, 12}^{\text {double }}-D_{v, 12}^{\text {single }}\right)$ and (b) the floor-wall transmission $\left(D_{v, 14}^{\text {double }}-D_{v, 13}^{\text {single }}\right)$.

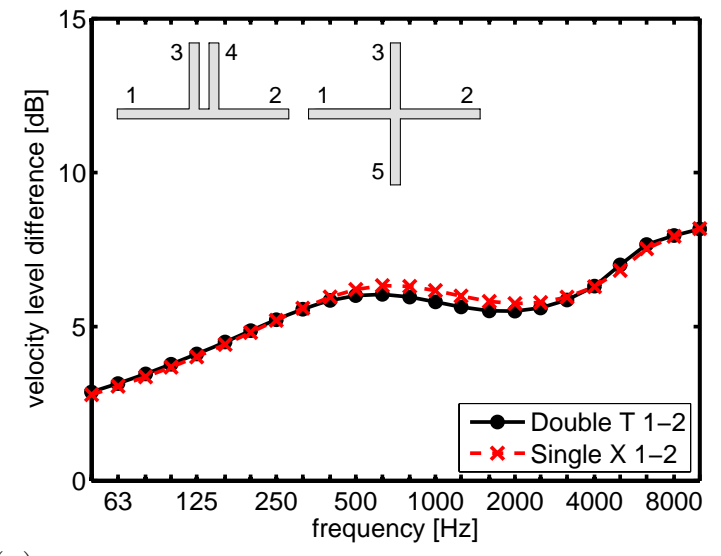

(a)

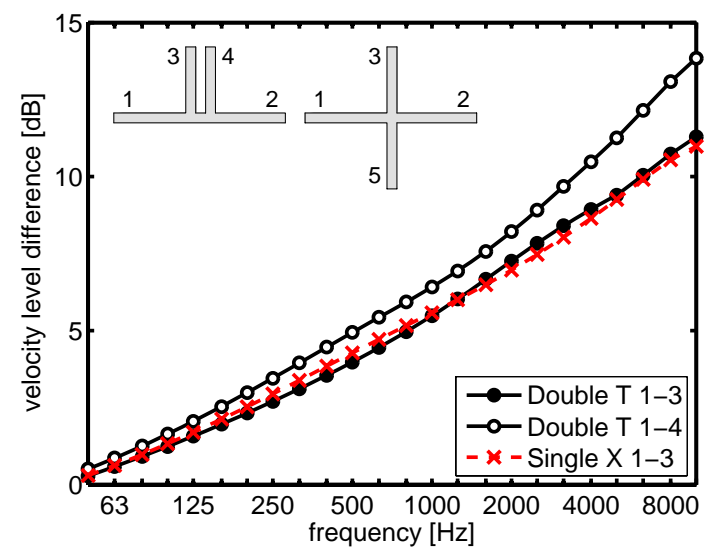

(b)

Figure 10: Velocity level difference for (i) a double wall T-junction with concrete floors $\left(h_{1}=h_{2}=0.15 \mathrm{~m}\right)$ and double concrete walls $\left(h_{3}=h_{4}=0.10 \mathrm{~m}, d=5 \mathrm{~cm}\right)$ or (ii) a single wall X-junction with concrete floors $\left(h_{1}=h_{2}=0.15 \mathrm{~m}\right)$ and concrete walls $\left(h_{3}=h_{5}=0.10 \mathrm{~m}\right)$ when plate 1 is excited. (a) Floor to floor transmission and (b) floor to wall transmission.

Figure 10 shows the velocity level difference for a T-junction with $15 \mathrm{~cm}$ concrete floors and a $10(5) 10 \mathrm{~cm}$ double concrete wall. The results are compared with the velocity level difference for a single wall $\mathrm{X}$-junction with $15 \mathrm{~cm}$ concrete floors and $10 \mathrm{~cm}$ concrete walls. The velocity level difference $D_{\mathrm{v}, 12}$ between floor and floor and $D_{\mathrm{v}, 13}$ between floor and first wall can be well predicted with an equivalent single wall $\mathrm{X}$-junction. The velocity level difference $D_{\mathrm{v}, 14}$ between floor and second wall is underestimated by the single wall model at high frequencies, but the agreement is still reasonable in a broad frequency range.

Figure 11 shows the velocity level difference for a X-junction with $10(5) 10 \mathrm{~cm}$ double concrete walls. 


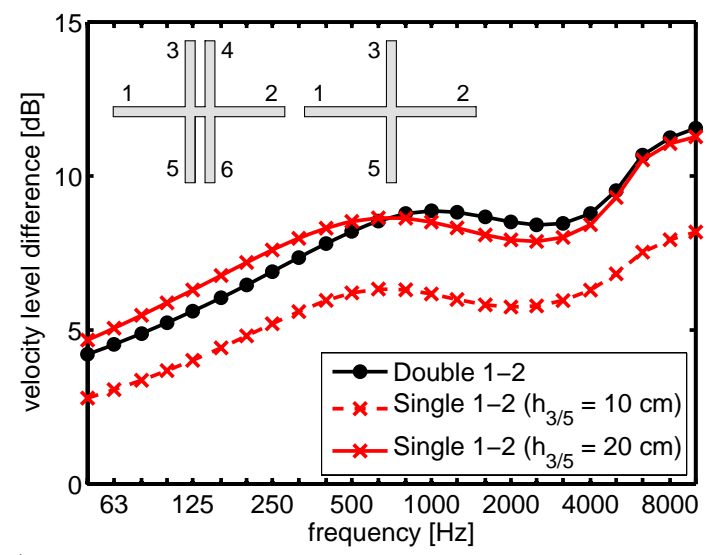

(a)

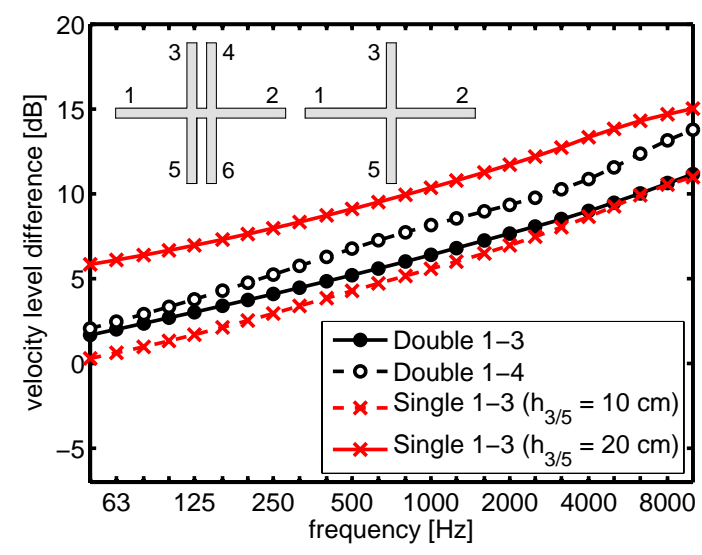

(b)

Figure 11: Velocity level difference for a X-junction with concrete floors $\left(h_{1}=h_{2}=0.15 \mathrm{~m}\right.$ ) and (i) double concrete walls $\left(h_{3}=h_{4}=h_{5}=h_{6}=0.10 \mathrm{~m}, d=5 \mathrm{~cm}\right.$ ), (ii) single concrete walls with $h_{3}=h_{5}=0.10 \mathrm{~m}$ or (iii) single concrete walls with $h_{3}=h_{5}=0.20 \mathrm{~m}$. (a) Floor to floor transmission and (b) floor to wall transmission.

The results are compared with the vibration reduction for two equivalent single wall junctions with $10 \mathrm{~cm}$ or $20 \mathrm{~cm}$ concrete walls. The floor-floor transmission path across the double walls $\left(D_{\mathrm{v}, 12}\right)$ can be well approximated by assuming an equivalent single wall with thickness $20 \mathrm{~cm}$. The vibration transmission from the floor to the first leaf is similar to that for a $\mathrm{X}$-junction with $10 \mathrm{~cm}$ thick single walls, especially at high frequencies where the two leafs of the double walls are effectively decoupled. The velocity level difference $D_{\mathrm{v}, 14}$ between the floor and the second wall cannot be accurately predicted with an equivalent single wall model. The floor-wall velocity level differences $D_{\mathrm{v}, 13}$ of the 10 and $20 \mathrm{~cm}$ single concrete wall models do give, however, a lower and upper limit, respectively.

\subsubsection{Structure-borne sound transmission across two adjacent single wall junctions}

By increasing the cavity width $d$, the double wall junction model can be used to investigate the limiting case of structure-borne sound transmission across two parallel single wall junctions. The effect of vibration coupling between two adjacent single wall junctions is investigated by comparing the coupled model of section 3 with a decoupled model [20]. In the decoupled model, the coupling plate is modeled as a SEA plate subsystem and the two junctions are considered independently. The coupling loss factors are determined from angular average transmission coefficients calculated with wave theory for single plate junctions [26]. The SEA matrix equations of the decoupled model can be found in appendix Appendix A.2.

Figure 12 compares the velocity level difference for a concrete plate junction as obtained with the coupled and decoupled SEA models. The $15 \mathrm{~cm}$ thick floors of the double T-junction have a width of $5 \mathrm{~m}$, the $10 \mathrm{~cm}$ thick walls have a height of $3.5 \mathrm{~m}$. In the coupled model, the SEA level difference is not smooth due to 


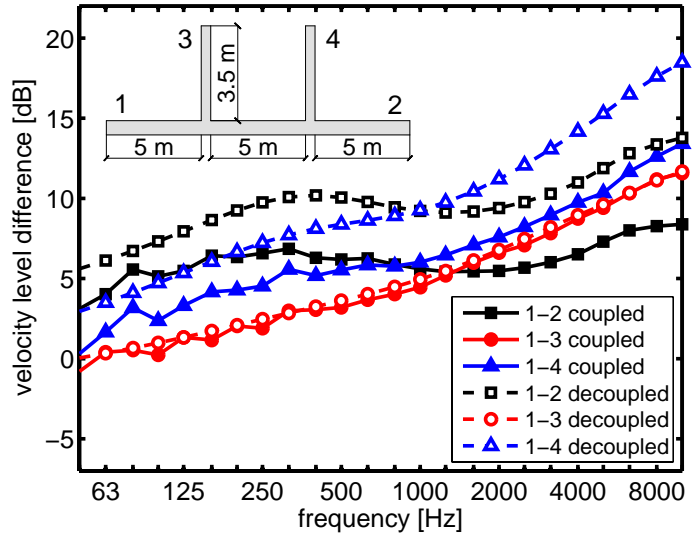

Figure 12: Velocity level difference for two adjacent T-junctions with concrete floors $\left(h_{1}=h_{2}=0.15 \mathrm{~m}\right)$ and concrete walls $\left(h_{3}=h_{4}=0.10 \mathrm{~m}\right)$. Coupled (solid lines) and decoupled (dotted lines) SEA model results.

fluctuations in the transmission coefficients caused by standing waves in the coupling plate which occur at certain angles of incidence [19, 20,23]. The vibration transmission from the floor to the first wall $\left(D_{\mathrm{v}, 13}\right)$ is not significantly altered when taking into account the coupling effect between the two junctions. The decoupled model underestimates, however, the vibration transmission to the second T-junction. The velocity level differences $D_{\mathrm{v}, 12}$ and $D_{\mathrm{v}, 14}$ are overestimated by $2.5 \mathrm{~dB}$ at low frequencies and more than $5 \mathrm{~dB}$ at high frequencies. This overestimation can be attributed to the tunneling effect which is present in chain-like plate assemblies [31]. Indirect coupling occurs between the bending wave subsystems of the first floor and the floor and wall of the second junction when the wavelength of in-plane waves is considerably larger than the dimensions of the intermediate plate. This is not accounted for in the decoupled model. Furthermore, the decoupled SEA model assumes a diffuse vibration field on the middle floor, whereas the angle-dependence of the transmission coefficients leads to spatial filtering and non-diffusiveness [32, 33]. The tunneling mechanisms become more important between distant subsystems in periodic structures like ribbed plates [32] or box-like structures [33].

\section{Conclusions}

In this paper, statistical models have been developed to predict the vibration transmission across rigid junctions composed of single and double walls. The coupling loss factors are calculated from angular average transmission coefficients using a wave approach for semi-infinite plates. It is shown that the in-plane wave transmission must be accounted for when modeling the structure-borne sound transmission across double wall junctions. Airborne coupling between the double wall leaves is disregarded, which is reasonable for heavyweight structures. The velocity level differences for double wall junctions show the same trends as for single wall junctions, but the vibration reduction for the double walls is about $1-3 \mathrm{~dB}$ larger in the case of 
heavyweight walls. Double wall T-junctions can be approximated by an equivalent single wall $\mathrm{X}$-junction. Double wall $\mathrm{X}$-junctions can be approximated by modeling the double walls with equivalent single walls. The thickness of the equivalent single wall should be either the thickness of a single leaf or the total thickness of both double wall leafs, depending on the transmission path of interest. Finally, the model has been used to assess the structure-borne sound transmission across two adjacent single wall junctions. When modeling the coupling plate between the two junctions as a separate SEA plate subsystem, the indirect coupling is neglected and the vibration transmission is underestimated.

\section{Acknowledgements}

Arne Dijckmans is a postdoctoral fellow of the Research Foundation Flanders (FWO). Part of this research was performed within a research stay at the Acoustics Research Unit of the University of Liverpool, funded by the FWO. The financial support is gratefully acknowledged.

\section{Appendix A. SEA equations for double wall junction}

\section{Appendix A.1. Coupled model}

For a double wall junction, following set of SEA equations can be set up:

$$
\left[\begin{array}{cc}
{\left[\alpha^{(1)}\right]} & {\left[\beta^{(2)(1)}\right]} \\
{\left[\beta^{(1)(2)}\right]} & {\left[\alpha^{(2)}\right]}
\end{array}\right]\left[\begin{array}{l}
{\left[E^{(1)}\right]} \\
{\left[E^{(2)}\right]}
\end{array}\right]=\frac{1}{\omega}\left[\begin{array}{l}
{\left[W_{\text {in }}^{(1)}\right]} \\
{\left[W_{\text {in }}^{(2)}\right]}
\end{array}\right],
$$

with

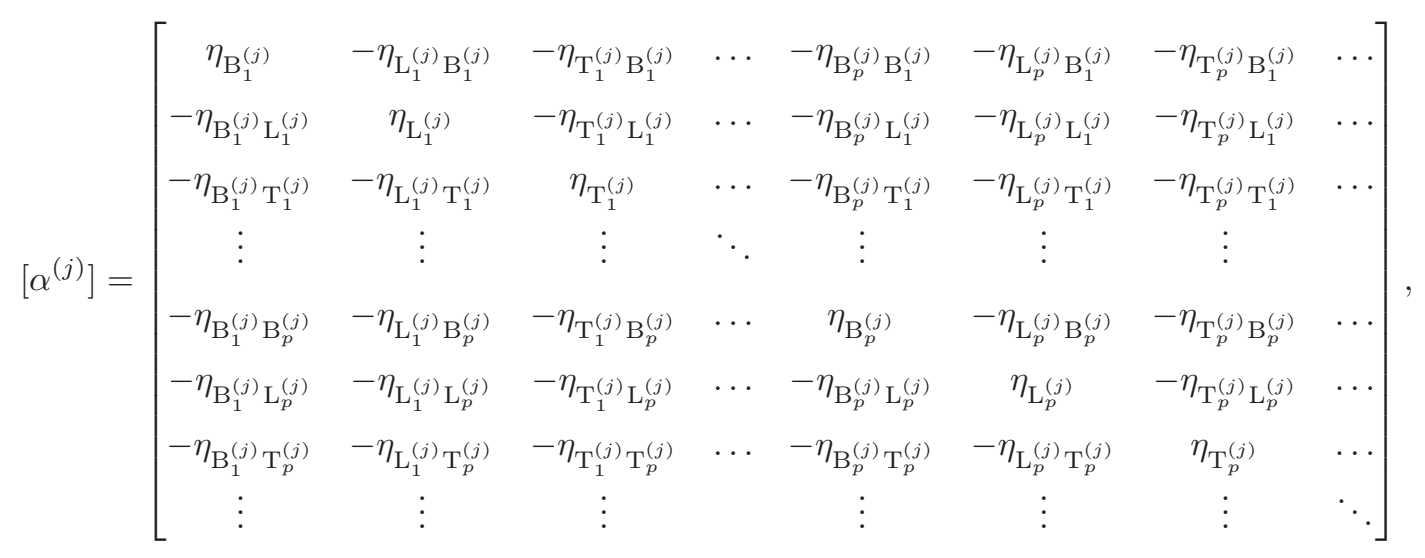




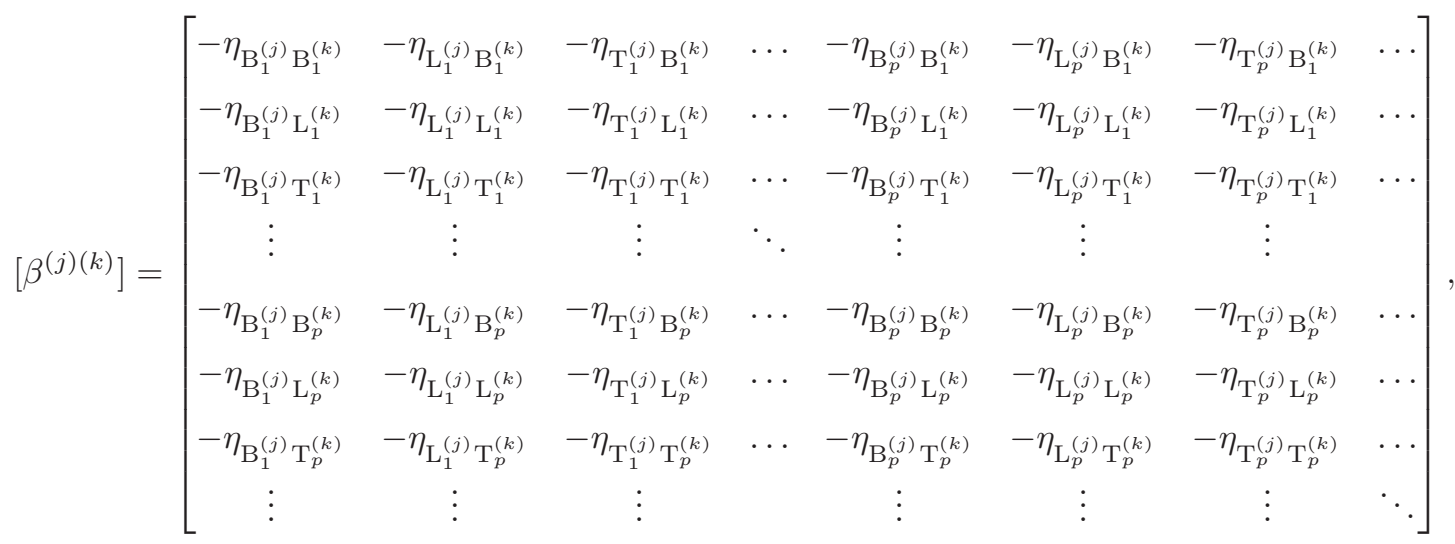

$$
\begin{aligned}
& {\left[E^{(j)}\right]=\left[E_{\mathrm{B}_{1}^{(j)}} E_{\mathrm{L}_{1}^{(j)}} E_{\mathrm{T}_{1}^{(j)}} \cdots E_{\mathrm{B}_{p}^{(j)}} E_{\mathrm{L}_{p}^{(j)}} E_{\mathrm{T}_{p}^{(j)}} \cdots\right]^{T},}
\end{aligned}
$$

and

$$
\left[W_{\mathrm{in}}^{(j)}\right]=\left[W_{\mathrm{B}_{1}^{(j)}} W_{\mathrm{L}_{1}^{(j)}} W_{\mathrm{T}_{1}^{(j)}} \cdots W_{\mathrm{B}_{p}^{(j)}} W_{\mathrm{L}_{p}^{(j)}} W_{\mathrm{T}_{p}^{(j)}} \cdots\right]^{T}
$$

The coupling loss factors $\eta_{i j}$ between different subsystems (B: bending wave, L: quasi-longitudinal wave, T: transverse wave) are calculated from angular average transmission coefficients from the wave theory for double wall junctions by means of equation (105). $E_{\mathrm{B}_{p}^{(j)}}, E_{\mathrm{L}_{p}^{(j)}}$, and $E_{\mathrm{T}_{p}^{(j)}}$ are the bending, quasi-longitudinal and in-plane transverse wave energy of plate $p$ of junction $j$, respectively. $W_{\mathrm{B}_{p}^{(j)}}, W_{\mathrm{L}_{p}^{(j)}}$, and $W_{\mathrm{T}_{p}^{(j)}}$ are the power input in the respective subsystem. For the case of a vertical excitation on plate $p=1$ of junction $j=1$ considered in this paper, only the power input $W_{\mathrm{B}_{1}^{(1)}}$ is different from zero. The diagonal elements of matrix $\left[\alpha^{(j)}\right]$ are determined by

$$
\begin{aligned}
& \eta_{\mathrm{B}_{p}^{(1)}}=\eta_{p}^{(1)}+\sum_{n \neq p}^{N^{(1)}}\left(\eta_{\mathrm{B}_{p}^{(1)} \mathrm{B}_{n}^{(1)}}+\eta_{\mathrm{B}_{p}^{(1)} \mathrm{L}_{n}^{(1)}}+\eta_{\mathrm{B}_{p}^{(1)} \mathrm{T}_{n}^{(1)}}\right)+\sum_{n=1}^{N^{(2)}}\left(\eta_{\mathrm{B}_{p}^{(1)} \mathrm{B}_{n}^{(2)}}+\eta_{\mathrm{B}_{p}^{(1)} \mathrm{L}_{n}^{(2)}}+\eta_{\mathrm{B}_{p}^{(1)} \mathrm{T}_{n}^{(1)}}\right), \\
& \eta_{\mathrm{T}_{p}^{(1)}}=\eta_{p}^{(1)}+\sum_{n \neq p}^{N^{(1)}}\left(\eta_{\mathrm{T}_{p}^{(1)} \mathrm{B}_{n}^{(1)}}+\eta_{\mathrm{T}_{p}^{(1)} \mathrm{L}_{n}^{(1)}}+\eta_{\mathrm{T}_{p}^{(1)} \mathrm{T}_{n}^{(1)}}\right)+\sum_{n=1}^{N^{(2)}}\left(\eta_{\mathrm{T}_{p}^{(1)} \mathrm{B}_{n}^{(2)}}+\eta_{\mathrm{T}_{p}^{(1)} \mathrm{L}_{n}^{(2)}}+\eta_{\mathrm{T}_{p}^{(1)} \mathrm{T}_{n}^{(2)}}\right), \\
& \eta_{\mathrm{L}_{p}^{(1)}}=\eta_{p}^{(1)}+\sum_{n \neq p}^{N^{(1)}}\left(\eta_{\mathrm{L}_{p}^{(1)} \mathrm{B}_{n}^{(1)}}+\eta_{\mathrm{L}_{p}^{(1)} \mathrm{L}_{n}^{(1)}}+\eta_{\mathrm{L}_{p}^{(1)} \mathrm{T}_{n}^{(1)}}\right)+\sum_{n=1}^{N^{(2)}}\left(\eta_{\mathrm{L}_{p}^{(1)} \mathrm{B}_{n}^{(2)}}+\eta_{\mathrm{L}_{p}^{(1)} \mathrm{L}_{n}^{(2)}}+\eta_{\mathrm{L}_{p}^{(1)} \mathrm{T}_{n}^{(2)}}\right), \\
& \eta_{\mathrm{B}_{p}^{(2)}}=\eta_{p}^{(2)}+\sum_{n=1}^{N^{(1)}}\left(\eta_{\mathrm{B}_{p}^{(2)} \mathrm{B}_{n}^{(1)}}+\eta_{\mathrm{B}_{p}^{(2)} \mathrm{L}_{n}^{(1)}}+\eta_{\mathrm{B}_{p}^{(2)} \mathrm{T}_{n}^{(1)}}\right)+\sum_{n \neq p}^{N^{(2)}}\left(\eta_{\mathrm{B}_{p}^{(2)} \mathrm{B}_{n}^{(2)}}+\eta_{\mathrm{B}_{p}^{(2)} \mathrm{L}_{n}^{(2)}}+\eta_{\mathrm{B}_{p}^{(2)} \mathrm{T}_{n}^{(1)}}\right), \\
& \eta_{\mathrm{T}_{p}^{(2)}}=\eta_{p}^{(2)}+\sum_{n=1}^{N^{(1)}}\left(\eta_{\mathrm{T}_{p}^{(2)} \mathrm{B}_{n}^{(1)}}+\eta_{\mathrm{T}_{p}^{(2)} \mathrm{L}_{n}^{(1)}}+\eta_{\mathrm{T}_{p}^{(2)} \mathrm{T}_{n}^{(1)}}\right)+\sum_{n \neq p}^{N^{(2)}}\left(\eta_{\mathrm{T}_{p}^{(2)} \mathrm{B}_{n}^{(2)}}+\eta_{\mathrm{T}_{p}^{(2)} \mathrm{L}_{n}^{(2)}}+\eta_{\mathrm{T}_{p}^{(2)} \mathrm{T}_{n}^{(2)}}\right), \\
& \eta_{\mathrm{L}_{p}^{(2)}}=\eta_{p}^{(2)}+\sum_{n=1}^{N^{(1)}}\left(\eta_{\mathrm{L}_{p}^{(2)} \mathrm{B}_{n}^{(1)}}+\eta_{\mathrm{L}_{p}^{(2)} \mathrm{L}_{n}^{(1)}}+\eta_{\mathrm{L}_{p}^{(2)} \mathrm{T}_{n}^{(1)}}\right)+\sum_{n \neq p}^{N^{(2)}}\left(\eta_{\mathrm{L}_{p}^{(2)} \mathrm{B}_{n}^{(2)}}+\eta_{\mathrm{L}_{p}^{(2)} \mathrm{L}_{n}^{(2)}}+\eta_{\mathrm{L}_{p}^{(2)} \mathrm{T}_{n}^{(2)}}\right),
\end{aligned}
$$


where $\eta_{p}^{(j)}$ is the internal loss factor. It is assumed that the internal loss factor for bending, quasi-longitudinal and in-plane transverse wave propagation is the same.

\section{Appendix A.2. Decoupled model}

When the coupling plate is modeled as a SEA plate subsystem and the two junctions are considered independently as two single wall junctions, following set of SEA equations can be set up:

$$
\left[\begin{array}{ccc}
{\left[\alpha^{(1)}\right]} & {[0]} & {\left[\gamma^{(1)}\right]} \\
{[0]} & {\left[\alpha^{(2)}\right]} & {\left[\gamma^{(2)}\right]} \\
{\left[\delta^{(1)}\right]} & {\left[\delta^{(2)}\right]} & {[\epsilon]}
\end{array}\right]\left[\begin{array}{c}
{\left[E^{(1)}\right]} \\
{\left[E^{(2)}\right]} \\
{\left[E^{(12)}\right]}
\end{array}\right]=\frac{1}{\omega}\left[\begin{array}{c}
{\left[W_{\text {in }}^{(1)}\right]} \\
{\left[W_{\text {in }}^{(2)}\right]} \\
{\left[W_{\text {in }}^{(12)}\right]}
\end{array}\right]
$$

with $\left[\alpha^{(j)}\right]$ as defined in Eq. (A.2), $\left[E^{(j)}\right]$ as defined in Eq. (A.4), $\left[W_{\text {in }}^{(j)}\right]$ as defined in Eq. (A.5),

$$
\begin{aligned}
& {\left[\gamma^{(j)}\right]=\left[\begin{array}{ccc}
-\eta_{\mathrm{B}^{(12)} \mathrm{B}_{1}^{(j)}} & -\eta_{\mathrm{L}^{(12)} \mathrm{B}_{p}^{(j)}} & -\eta_{\mathrm{T}^{(12)} \mathrm{B}_{1}^{(j)}} \\
-\eta_{\mathrm{B}^{(12)} \mathrm{L}_{1}^{(j)}} & -\eta_{\mathrm{L}^{(12)} \mathrm{L}_{p}^{(j)}} & -\eta_{\mathrm{T}^{(12)} \mathrm{L}_{1}^{(j)}} \\
-\eta_{\mathrm{B}^{(12)} \mathrm{T}_{1}^{(j)}} & -\eta_{\mathrm{L}^{(12)} \mathrm{T}_{p}^{(j)}} & -\eta_{\mathrm{T}^{(12)} \mathrm{T}_{1}^{(j)}} \\
\vdots & \vdots & \vdots \\
-\eta_{\mathrm{B}^{(12)} \mathrm{B}_{p}^{(j)}} & -\eta_{\mathrm{L}^{(12)} \mathrm{B}_{p}^{(j)}} & -\eta_{\mathrm{T}^{(12)} \mathrm{B}_{p}^{(j)}} \\
-\eta_{\mathrm{B}^{(12)} \mathrm{L}_{p}^{(j)}} & -\eta_{\mathrm{L}^{(12)} \mathrm{L}_{p}^{(j)}} & -\eta_{\mathrm{T}^{(12)} \mathrm{L}_{p}^{(j)}} \\
-\eta_{\mathrm{B}_{(12)} \mathrm{T}_{p}^{(j)}} & -\eta_{\mathrm{L}_{(12)} \mathrm{T}_{p}^{(j)}} & -\eta_{\mathrm{T}^{(12)} \mathrm{T}_{p}^{(j)}} \\
\vdots & \vdots & \vdots
\end{array}\right]} \\
& {\left[\delta^{(j)}\right]=\left[\begin{array}{cccccccc}
-\eta_{\mathrm{B}_{1}^{(j)} \mathrm{B}^{(12)}} & -\eta_{\mathrm{L}_{1}^{(j)} \mathrm{B}^{(12)}} & -\eta_{\mathrm{T}_{1}^{(j)} \mathrm{B}^{(12)}} & \cdots & -\eta_{\mathrm{B}_{p}^{(j)} \mathrm{B}^{(12)}} & -\eta_{\mathrm{L}_{p}^{(j)} \mathrm{B}^{(12)}} & -\eta_{\mathrm{T}_{p}^{(j)} \mathrm{B}^{(12)}} & \cdots \\
-\eta_{\mathrm{B}_{1}^{(j)} \mathrm{L}^{(12)}} & -\eta_{\mathrm{L}_{1}^{(j)} \mathrm{L}^{(12)}} & -\eta_{\mathrm{T}_{1}^{(j)} \mathrm{L}^{(12)}} & \cdots & -\eta_{\mathrm{B}_{p}^{(j)} \mathrm{L}^{(12)}} & -\eta_{\mathrm{L}_{p}^{(j)} \mathrm{L}^{(12)}} & -\eta_{\mathrm{T}_{p}^{(j)} \mathrm{L}^{(12)}} & \cdots \\
-\eta_{\mathrm{B}_{1}^{(j)} \mathrm{T}^{(12)}} & -\eta_{\mathrm{L}_{1}^{(j)} \mathrm{T}^{(12)}} & -\eta_{\mathrm{T}_{1}^{(j)} \mathrm{T}^{(12)}} & \cdots & -\eta_{\mathrm{B}_{p}^{(j)} \mathrm{T}^{(12)}} & -\eta_{\mathrm{L}_{p}^{(j)} \mathrm{T}_{1}^{(12)}} & -\eta_{\mathrm{T}_{p}^{(j)} \mathrm{T}^{(12)}} & \cdots
\end{array}\right],} \\
& {[\epsilon]=\left[\begin{array}{ccc}
\eta_{\mathrm{B}^{(12)}} & -\eta_{\mathrm{L}^{(12)} \mathrm{B}^{(12)}} & -\eta_{\mathrm{T}^{(12)} \mathrm{B}^{(12)}} \\
-\eta_{\mathrm{B}^{(12)} \mathrm{L}^{(12)}} & \eta_{\mathrm{L}^{(12)}} & -\eta_{\mathrm{T}^{(12)} \mathrm{L}^{(12)}} \\
-\eta_{\mathrm{B}^{(12)} \mathrm{T}^{(12)}} & -\eta_{\mathrm{L}^{(12)} \mathrm{T}^{(12)}} & \eta_{\mathrm{T}^{(12)}}
\end{array}\right]} \\
& {\left[E^{(12)}\right]=\left[E_{\mathrm{B}^{(12)}} E_{\mathrm{L}^{(12)}} E_{\mathrm{T}^{(12)}}\right]^{T},}
\end{aligned}
$$

and

$$
\left[W^{(12)}\right]=\left[W_{\mathrm{B}^{(12)}} W_{\mathrm{L}^{(12)}} W_{\mathrm{T}^{(12)}}\right]^{T} .
$$


The diagonal elements of matrix $[\epsilon]$ are determined by

$$
\begin{aligned}
& \eta_{\mathrm{B}^{(12)}}=\eta^{(12)}+\sum_{n=1}^{N^{(1)}}\left(\eta_{\mathrm{B}^{(12)} \mathrm{B}_{n}^{(1)}}+\eta_{\mathrm{B}^{(12)} \mathrm{L}_{n}^{(1)}}+\eta_{\mathrm{B}^{(12)} \mathrm{T}_{n}^{(1)}}\right)+\sum_{n=1}^{N^{(2)}}\left(\eta_{\mathrm{B}^{(12)} \mathrm{B}_{n}^{(2)}}+\eta_{\mathrm{B}^{(12)} \mathrm{L}_{n}^{(2)}}+\eta_{\mathrm{B}^{(12)} \mathrm{T}_{n}^{(1)}}\right) \\
& \eta_{\mathrm{T}^{(12)}}=\eta^{(12)}+\sum_{n=1}^{N^{(1)}}\left(\eta_{\mathrm{T}^{(12)} \mathrm{B}_{n}^{(1)}}+\eta_{\mathrm{T}^{(12)} \mathrm{L}_{n}^{(1)}}+\eta_{\mathrm{T}^{(12)} \mathrm{T}_{n}^{(1)}}\right)+\sum_{n=1}^{N^{(2)}}\left(\eta_{\mathrm{T}^{(12)} \mathrm{B}_{n}^{(2)}}+\eta_{\mathrm{T}^{(12)} \mathrm{L}_{n}^{(2)}}+\eta_{\mathrm{T}^{(12)} \mathrm{T}_{n}^{(2)}}\right) \\
& \eta_{\mathrm{L}^{(12)}}=\eta^{(12)}+\sum_{n=1}^{N^{(1)}}\left(\eta_{\mathrm{L}^{(12)} \mathrm{B}_{n}^{(1)}}+\eta_{\mathrm{L}^{(12)} \mathrm{L}_{n}^{(1)}}+\eta_{\mathrm{L}^{(12)} \mathrm{T}_{n}^{(1)}}\right)+\sum_{n=1}^{N^{(2)}}\left(\eta_{\mathrm{L}^{(12)} \mathrm{B}_{n}^{(2)}}+\eta_{\mathrm{L}^{(12)} \mathrm{L}_{n}^{(2)}}+\eta_{\mathrm{L}^{(12)} \mathrm{T}_{n}^{(2)}}\right)
\end{aligned}
$$

where $\eta^{(12)}$ is the internal loss factor of the coupling plate. The coupling loss factors are determined from angular average transmission coefficients calculated with wave theory for single wall $\mathrm{T}$ - or $\mathrm{X}$-junctions.

\section{References}

[1] International Organisation for Standardisation, ISO 15712:2005: Building acoustics - Estimation of acoustic performance of buildings from the performance of elements. Part 1: Airborne sound insulation between rooms. Part 2: Impact sound insulation between rooms. (2005).

[2] European Commmittee for Standardisation, EN 12354:2000: Building acoustics - Estimation of acoustic performance of buildings from the performance of elements. Part 1: Airborne sound insulation between rooms. Part 2: Impact sound insulation between rooms. (2000).

[3] E. Gerretsen, Calculation of the sound transmission between dwellings by partitions and flanking structures, Appl. Acoust. 12 (6) (1979) 413-433.

[4] C. Hopkins, Determination of vibration reduction indices using wave theory for junctions in heavyweight buildings, Acta Acust. united Ac. 100 (6) (2014) 1056-1066.

[5] C. Crispin, L. De Geetere, B. Ingelaere, Extensions of EN 12354 vibration reduction index expressions by means of FEM calculations, in: Proceedings of Inter-Noise 2014, Melbourne, Australia, 2014.

[6] S. Schoenwald, Flanking sound transmission through lightweight framed double leaf walls - prediction using statistical energy analysis, Ph.D. thesis, TU Eindhoven (2008).

[7] S. Schoenwald, B. Zeitler, I. Sabourin, Analysis on structure-borne sound transmission at junctions of solid wood double walls with continuous floors, in: Proceedings of 7th Forum Acusticum, Krakow, 2014.

[8] R. Craik, Sound Transmission through Buildings using Statistical Energy Analysis, Gower, England, 1996.

[9] L. Cremer, M. Heckl, B. A. T. Petersson, Structure-Borne Sound: Structural Vibrations and Sound Radiation at Audio Frequencies (3rd Edition), Springer, Berlin, 2005.

[10] S. Budrin, A. Nikiforov, Wave transmission through assorted plate joints, Soviet Physics-Acoustics 9 (4) (1964) 333-336.

[11] R. Lyon, E. Eichler, Random vibration of connected structures, J. Acoust. Soc. Am. 36 (7) (1964) 1344-1354.

[12] R. Langley, K. Heron, Elastic wave transmission through plate/beam junctions, J. Sound Vib. 143 (2) (1990) $241-253$.

[13] W. Whöle, T. Beckmann, H. Schreckenbach, Coupling loss factors for statistical energy analysis of sound transmission at rectangular structural slab joints. Parts I and II, J. Sound Vib. 77 (3) (1981) 323-344.

[14] P. Craven, B. Gibbs, Sound tranmsission and mode coupling at junctions of thin plates, Part I: Representation of the problem, J. Sound Vib. 77 (3) (1981) 417-427.

[15] M. McCollum, J. Cuschieri, Bending and in-plane wave transmission in thick connected plates using statistical energy analysis, J. Acoust. Soc. Am. 88 (3) (1990) 1480-1485. 
[16] P. Mees, G. Vermeir, Structure-borne sound transmission at elastically connected plates, J. Sound Vib. 166 (1) (1993) $55-76$.

[17] I. Bosmans, P. Mees, G. Vermeir, Structure-borne sound transmission between thin orthotropic plates: analytic solutions, J. Sound Vib. 191 (1) (1996) 75-90.

[18] I. Bosmans, G. Vermeir, Diffuse transmission of structure-borne sound at periodic junctions of semi-infinite plates, J. Acoust. Soc. Am. 101 (6) (1997) 3443-3456.

[19] M. Bhattacharya, K. Mulholland, M. Crocker, Propagation of sound energy by vibration transmission via structural junctions, J. Sound Vib. 18 (2) (1971) 221-234.

[20] R. Craik, R. Smith, Sound transmission through lightweight parallel plates. Part II: structure-borne sound, Appl. Acoust. 61 (2000) 247-269.

[21] R. Craik, R. Wilson, Sound transmission through parallel plates coupled along a line, Appl. Acoust. 49 (4) (1997) 353-372.

[22] R. Craik, T. Nightingale, J. Steel, Sound transmission through a double leaf partition with edge flanking, J. Acoust. Soc. Am. 101 (2) (1997) 964-969.

[23] B. Gibbs, Mode coupling and energy partition of sound in a system of plate junctions, J. Sound Vib. 104 (1) (1986) $127-136$.

[24] R. Craik, J. Steel, D. Evans, Statistical energy analysis of structure-borne sound transmission at low frequencies, J. Sound Vib. 144 (1) (1991) 95-107.

[25] F. Fahy, A. Mohammed, A study of the uncertainty in applications of SEA to coupled beam and plate systems, part 1: Computational experiments, J. Sound Vib. 158 (1) (1992) 45-67.

[26] C. Hopkins, Sound insulation, Elsevier Ltd., Oxford, 2007.

[27] C. Hopkins, Statistical energy analysis of coupled plate systems with low modal density and low modal overlap, J. Sound Vib. 251 (2) (2002) 193-214.

[28] A. Dijckmans, Structure-borne sound transmission across junctions of finite single and double walls, in: Proceedings of Inter-Noise 2015, the 2015 International Congress and Exposition on Noise Control Engineering, San Francisco, California, USA, 2015.

[29] I. Bosmans, Analytical modelling of structure-borne sound transmission and modal interaction at complex plate junctions, Ph.D. thesis, KU Leuven (1998).

[30] B. Van Genechten, O. Atak, B. Bergen, E. Deckers, S. Jonckheere, J. Lee, A. Maressa, K. Vergote, B. Pluymers, D. Vandepitte, W. Desmet, An efficient wave based method for solving helmholtz problems in three-dimensional bounded domains, Engineering Analysis with Boundary Elements 36 (2012) 63-75.

[31] K. Heron, Advanced statistical energy analysis, Philosophical Transactions of the Royal Society of London A 346 (1681) (1994) 501-510.

[32] J. Yin, C. Hopkins, Prediction of high-frequency vibration transmission across coupled, periodic ribbed plates by incorporating tunneling mechanisms, J. Acoust. Soc. Am. 133 (4) (2013) 2069-2081.

[33] D. Wilson, C. Hopkins, Analysis of bending wave transmission using beam tracing with advanced statistical energy analysis for periodic box-like structures affected by spatial filtering, J. Sound Vib. 341 (2015) 138-161. 